\title{
SAR Raw Signal Simulation of Oil Slicks in Ocean Environments
}

\author{
Giorgio Franceschetti, Life Fellow, IEEE, Antonio Iodice, Member, IEEE, Daniele Riccio, Senior Member, IEEE, \\ Giuseppe Ruello, Student Member, IEEE, and Roberta Siviero
}

\begin{abstract}
Synthetic aperture radar (SAR) raw signal simulation is a powerful tool for design of oil slick detection and interpretation systems. In this paper, the ocean simulation issues are presented, and the main problems relating to the oil presence on the sea surface are treated. Attention is focused on the electromagnetic side of the problem, with care to the sensor signatures, the dielectric, physical-chemical, and geometric nature of the oil slick, and to the environmental conditions. The presented SAR simulator is based on an ocean model and an oil slick model. The former makes use of multiscale description of the ocean surface: the distributed surface model for the SAR-ocean interaction is considered by taking into account the nonlinear hydrodynamic effect for the water particle movement. The latter model implements a modification of the ocean spectrum, based on the Marangoni theory and accounting for the nonlinear wave interaction mechanism. However, the proposed SAR raw signal simulator is modular and flexible, thus allowing other possible physical models for modeling the oil slick effect over the ocean spectrum. Meaningful SAR simulation experiments are presented and discussed, elucidating the role of difference on pollutants, oil thickness, wind speed and direction, incident wavelength and angle and other radar parameters. Validation of the simulator is also presented by comparison with experimental data. A striking conclusion of the paper is that higher order moments (from the second on) of oil slick SAR image statistics are quite different compared to those pertinent to an equivalent wind speed decrease on the imaged area. This suggests a convenient way to define new appropriate oil slick signatures.
\end{abstract}

Index Terms-Oceans, sea surface electromagnetic scattering, simulation, synthetic aperture radar (SAR), water pollution.

\section{INTRODUCTION}

$\mathbf{T}$ HE OCEAN covers about $70 \%$ of the earth's surface, and it plays a relevant role in the complex mechanisms that regulate the earth's environment. It is in continuous interaction with the atmosphere and the lithosphere, contributing to the global climate regulation, to the carbon and water cycles, and, last but not least, is a great resource for mankind.

Human activities are changing the ocean and its delicate balance. One of the most important causes of pollution in the ocean is the intensive oil emission onto the water. Natural oil is present in the ocean due to natural phenomena, but many statistic studies showed that human activities change the percentage of oil in water, sometimes with dramatic effects [1]. Oil spills caused by

Manuscript received July 3, 2001; revised July 12, 2002.

G. Franceschetti, A. Iodice, D. Riccio, and G. Ruello are with the Dipartimento di Ingegneria Elettronica e delle Telecomunicazioni, Università di Napoli "Federico II", 80125 Naples, Italy (e-mail: gfrance@unina.it; iodice@ unina.it; daniele.riccio@unina.it; ruello@unina.it).

R. Siviero is with Wide Sensing (WISE), 80078 Pozzuoli (NA), Italy.

Digital Object Identifier 10.1109/TGRS.2002.803798 accidental or intentional emissions interact with the local marine ecosystems and, by modifying the delicate air-sea balance, exhibit great influence on large- and short-scale phenomena.

The effects of oil pollution on the ocean have been widely studied by different viewpoints (chemical, physical, biological), with several methods (theoretical and experimental studies, "ad hoc" missions) by using several sensors (optical, microwave, airborne, and spaceborne), for both prevention and control objectives.

Remote sensing has proved to be a powerful tool to study ocean dynamics and detection of oil spills. Optical, radiometric, and radar systems have been employed for the detection of oil slicks in the sea. In particular, synthetic aperture radar (SAR) systems have been shown to be very useful. As a matter of fact, the main effect of oil on the ocean surface consists of dampening the capillary waves: this reduces electromagnetic backscattering from areas where oil is present and, therefore, generates darker zones in the SAR image, thus allowing, in principle, oil spill detection [2], [3]. Unfortunately, dark areas in SAR images can be caused by several other phenomena (presence of fitoplankton, algae, internal waves) [4], constituting a reason of possible ambiguity. Besides, lack of wind does not create capillary waves in the oil-free areas, making it impossible to detect the slick, whereas too intense wind spreads the slicks quickly and creates a clutter that makes the slick detection more difficult [5], [6]. Accordingly, algorithms that identify dark areas and separate those caused by oil slicks from others are not easy to be designed: to this aim, a SAR raw signal simulator that allows to quantitatively predict the effect of oil slicks on SAR images is highly useful. The simulator should rely on a sound physical model that allows evaluating the SAR return starting from knowledge of sea state and oil properties. To the best of our knowledge, such a model is not yet available. In fact, a huge amount of studies on oil slick effect on SAR images can be found in literature, but most of them consist of measurements over SAR images relevant to oil slicks in the presence of different sea states, and no quantitative relation is given between oil slick properties and the corresponding SAR signal.

In this paper, we outline a physical model of interaction between the oil-covered sea surface and the electromagnetic wave, and we explore the influence on SAR images of the presence of oil on the sea surface by using a SAR raw data simulator developed for ocean scenes [7]. An oceanographic model that predicts sea surface modifications caused by an oil layer [8] is chosen and used, and the effect of the oil layer over the electromagnetic properties of the surface is analyzed in detail. The simulator generates the corresponding SAR raw signal by using 
an appropriate SAR model that accounts for the ocean dynamics and for the electromagnetic wave-ocean surface interaction. It is important to emphasize that the quoted simulator generates the SAR raw signal and not the SAR image: simulation of the SAR raw signal is highly desirable whenever SAR focusing procedures are to be proposed, tested, and developed. To the best of the authors' knowledge, this is the only SAR raw signal simulator for ocean scenes available in the open literature.

The paper is organized as follows. Section II recalls the main concepts of ocean surface modeling, and Section III is concerned about the SAR raw signal simulation procedure [7]. The sea surface is divided into facets. The electromagnetic return from each facet is evaluated by focusing our attention on the Bragg resonance effect that, as commonly accepted in literature, is the dominant scattering mechanism for marine scenes at intermediate incidence angles [2], [3], [9]. It must be noted that sea surface movements during the SAR integration time are by no means negligible, so that they appreciably affect SAR imagery. The ocean dynamic effect on SAR images has been a very controversial issue. Phase velocity of long and short waves and orbital movements of particles must be considered. We make use of the distributed surface (DS) model [7], [10]-[13] to perform the simulation.

Section IV deals with the effect of the presence of oil over sea surface properties. A model, employing the Marangoni theory and a nonlinear energy transfer theory, is used to relate sea surface spectrum modifications to wind speed and physical-chemical slick properties.

Section V evaluates the effects of both the geometrical and dielectric parameters of the slick on the SAR imagery formation mechanisms. We show that usually the oil dielectric constant does not significantly modify the electromagnetic return, while the dominant effect is played by the dampening effect of oil on the capillary waves.

Section VI presents some examples of simulation. The main aim of such a simulation is to appreciate the backscattered return via qualitative and quantitative considerations, with only the physical description of the scene as input. A comparison between lack of wind and oil effects on SAR images is also presented by making reference to available physical models. Quantitative norms are introduced on SAR images: dampening and normalized intensity moments. These norms are evaluated for SAR simulated images at different bands, whenever oil is present; quantitative comparison against oil-free conditions is also reported with the important result that variance and the additional intensity moments allow a precise signature of the oil slick. Finally, conclusions are reported in Section VII.

\section{CONCEPTS OF OCEAN MODELING}

Oceans are nonlinear dynamic systems, whose physics is governed by very complex laws. Description of ocean surface waves is the most relevant branch of oceanography for electromagnetic sea sensing.

Surface waves are found in the ocean with wavelengths ranging from the hundred of meters to the millimeter scale [3]. The ocean wave range is usually divided into long (several hundred meters length), intermediate (tens of meters length), and short (less than one meter) waves [14]-[17]. In the literature, the ocean waves are also classified according to the physical phenomenon that dominates their formation: long and intermediate waves are usually referred to as gravity waves; conversely, the range of short waves includes the shortest gravity waves, and gravity-capillary (one centimeter to decimeters) and capillary (less than $1 \mathrm{~cm}$ ) waves [14]-[17].

Loosely speaking, when the wind blows over the ocean surface, capillary waves rise first. Then, the capillary waves transfer energy to waves with longer wavelength. This phenomenon continues until equilibrium is reached (fully developed sea) [2], [3]. Such an equilibrium depends on the wind strength. When the wind stops blowing, the short waves decrease quickly, while the long waves propagate far from their source (and, in this case, are usually referred to as swell [18]). Accordingly, long waves are generated by strong far winds, at variance of short waves, which are generated by local winds. It can be concluded that their directions of propagation are usually different and that they interact in a very complex way. Besides, it has been demonstrated that intermediate and long waves modulate the amplitude of the short waves [19], [20].

This involved physics is usually described (at least as long as remote sensing applications are concerned) by means of a sea surface energy spectrum [2], [21], [22], and of the following classical dispersion relation [2]

$$
\omega^{2}=g \kappa+\frac{\tau}{\rho} \kappa^{3}
$$

where $\boldsymbol{\omega}$ is the angular frequency of the ocean wave; $g\left[\mathrm{~m} / \mathrm{s}^{2}\right]$ is the gravity acceleration; $\tau[\mathrm{N} / \mathrm{m}]$ is the surface tension; $\rho$ $\left[\mathrm{kg} / \mathrm{m}^{3}\right]$ is the water density; and $\kappa=\left(\boldsymbol{\kappa}_{x}^{2}+\boldsymbol{\kappa}_{y}^{2}\right)^{1 / 2}[1 / \mathrm{m}]$ is the surface wavenumber. The dispersion equation (2.1) clearly shows that long waves are governed by the gravity force, at variance of very short waves, related to the surface tension.

An exhaustive discussion on problems involved in the whole ocean spectrum definition, use, and meaning is beyond the aim of this paper and can be found in the specialized literature [14]-[17]. We emphasize, hereafter, only shape and signatures of some common spectra and their relevance to electromagnetic studies and SAR image formation, as detailed in the Section III.

\section{OCEAN ScEnE Simulation PRocedure}

\section{A. SAR Raw Signal Simulation}

As stated in Section I, a SAR raw signal simulator is a convenient tool to study oil slick modification of the ocean SAR signature. In this paper, we make use of a SAR raw signal simulator [7] that accounts for most of the complex relations that play a significant role in the problem. In this simulator, we consider both geometrical and sensor parameters, the dielectric nature of the surface, the ocean long waves, the tilt modulation, and the ocean surface microstructure. This SAR raw signal simulator was tested and verified against canonical as well as experimental data for clean water [7]. We recall, hereafter, the rationale of this simulator in order to eventually identify key points to be modified to account for the oil spill presence (see Section V).

Let $t$ be the independent time variable and $x$ and $r$ be the independent space variables standing, respectively, for azimuth 
and range. By using prime coordinates for the SAR raw signal, this can be expressed as [7]

$$
s\left(x^{\prime}, r^{\prime}\right)=\iint d x d r \gamma\left(x, r, x^{\prime}=v_{s} t\right) g\left(x^{\prime}-x, r^{\prime}-r ; r\right)
$$

where $\gamma\left(x, r, x^{\prime}\right)$ is the (time-varying) reflectivity pattern of the ocean; $v_{s}$ is the sensor velocity; and $g\left(x^{\prime}-x, r^{\prime}-r ; r\right)$ is the unit impulse response of the SAR system.

Time dependence of the reflectivity function accounts for ocean waves dynamics and makes evaluation of the raw signal not immediate. In our simulator, the Harger DS model is used. According to this model, the time dependence can appropriately "migrate" from the reflectivity function to the impulsive response function provided that a dispersion relation for the long wave spectrum is available: this is the case for the swell system [7], [10]-[12]. Hence, for a swell system and in the frame of the DS model [10]-[12], we get [7]

$$
\begin{aligned}
& s\left(x^{\prime}, r^{\prime}\right)=\frac{1}{(2 \pi)^{2}} \exp \left\{j\left[\boldsymbol{\omega}_{0}-\boldsymbol{\omega}_{\xi} \xi_{0}-\boldsymbol{\omega}_{\eta} \eta_{0}\right] \frac{x^{\prime}}{v_{s}}\right\} \\
& \cdot s_{0}\left[\left(1+\frac{\boldsymbol{\omega}_{\xi}}{v_{s}}\right) x^{\prime}, r^{\prime}+\frac{\boldsymbol{\omega}_{\eta}}{v_{s}} x^{\prime}\right]
\end{aligned}
$$

wherein

$$
s_{0}\left(x^{\prime}, r^{\prime}\right)=\iint d x d r \gamma\left(x, r, x^{\prime}=0\right) g\left(x^{\prime}-x, r^{\prime}-r ; r\right)
$$

$\boldsymbol{\omega}=\boldsymbol{\omega}(\xi, \eta)$ is the long wave dispersion relation; $\xi=\kappa_{x}, \eta=\kappa_{y} / \sin \vartheta$ with $\vartheta$ being the SAR look angle; $\boldsymbol{\omega}_{\xi}=\partial \boldsymbol{\omega} /\left.\partial \xi\right|_{\xi=\xi_{0}, \eta=\eta_{0}}, \boldsymbol{\omega}_{\eta}=\partial \boldsymbol{\omega} /\left.\partial \boldsymbol{\eta}\right|_{\xi=\xi_{0}, \eta=\eta_{0}}$ with $\xi_{0}$, $\boldsymbol{\eta}_{0}$ being the wavenumbers corresponding to the dominant wavelength of the long wave spectrum; and $\omega_{0}$ is the related angular frequency. Accordingly, the raw signal $s_{0}(\cdot)$ is first computed via the fast Fourier transform method described in [23] and [24]; then, the final raw signal $s(\cdot)$ is recovered via a proper interpolation, as detailed in [7].

Evaluation of ocean surface and its reflectivity pattern (at a fixed time) is now in order.

\section{B. Ocean Surface Description}

Our simulator makes use of a multiscale model of the sea surface: long waves are modeled by a macroscopic profile, approximated by planar facets smaller than the SAR resolution, but much larger than the electromagnetic wavelength; a microscopic random roughness, with the proper power spectrum (i.e., a random collection of gravity-capillary and capillary wave patterns) is superimposed to these facets, in order to account for the short waves. This model is justified by the fact that typical SAR system resolutions are very small with respect to long waves, but very large with respect to short waves. The intermediate wave effect is included by adding a random tilting to the facet mean plane [19], [20].

The long wave is generated as a realization of a random process describing a swell profile at a fixed time: central wavenumber, amplitude, and bandwidth of its spectrum are input parameters of the simulator. They depend on the far wind intensity and direction and on the fetch area [18]. The algorithm for the long wave generation is detailed in [25].
With regard to short waves, we consider the Pierson-Moskovitz spectrum [2]. The directional spectrum $W(\boldsymbol{\kappa})$ is usually expressed as the product $W(\boldsymbol{\kappa})=S(\boldsymbol{\kappa}) f(\boldsymbol{\kappa}, \phi)$ where $f(\kappa, \phi)$ is the spreading function, and $\phi$ is defined by

$$
\left\{\begin{array}{l}
\kappa_{x}=\kappa \cos \phi \\
\kappa_{y}=\kappa \sin \phi
\end{array} .\right.
$$

The spreading function $f(\cdot)$ is usually practically constant with $\kappa$, whereas dependence on $\phi$ is of a cosine type [26] possibly raised to an appropriate exponent. With regard to $S(\kappa)$, short gravity range exhibits a power law shape [2], whereas gravitycapillary $S_{g c}(\kappa)$ and capillary $S_{c}(\kappa)$ ranges exhibit the shapes [2]

$$
S_{g c}(\kappa)=A_{m} \Omega_{m}^{p-1} \frac{\frac{g+3 g \kappa^{2}}{K_{m}^{2}}}{\left(\frac{g \kappa+g \kappa^{3}}{K_{m}^{2}}\right)^{(p+1) / 2}}
$$

and

$$
S_{c}(\kappa)=\frac{u_{*}^{3}}{U_{m}^{3}} \frac{K_{m}^{6}}{\kappa^{9}}
$$

respectively, with $K_{m}=\sqrt{g \rho / \tau}, A_{m}=0.875 \times 10^{-4}\left[\mathrm{~m}^{2}\right]$, $U_{m}=0.1893[\mathrm{~m} / \mathrm{s}]$, and $\Omega_{m}=2 \pi\left[\mathrm{s}^{-1}\right]$. In (3.5)-(3.6), $u_{*}$ is the friction velocity, related to wind speed and surface properties (see Section IV), and $p=5.0-\log _{10}\left(u_{*} / u_{0}\right)$, with $u_{0}=0.01 \mathrm{~m} / \mathrm{s}$. In particular, $S_{g c}(\kappa)$ is employed for surface wavenumbers ranging from $\kappa=90 \mathrm{~m}^{-1}$ (i.e., waves with wavelength of $7 \mathrm{~cm}$ ) up to the intersection point with $S_{c}(\kappa)$, beyond which surface tension is the dominant force and $S_{c}(\kappa)$ applies. In this model, the intersection point depends on the friction velocity, thus on the local wind according to (3.5) and (3.6). For wind speed (at a 10-m height) ranging from $4-9 \mathrm{~m} / \mathrm{s}$, the intersection point ranges from $740-895 \mathrm{~m}^{-1}$ (i.e., wavelength from $7-8.5 \mathrm{~mm}$ ).

Summarizing the above discussion, we can state that the surface profile within a facet is

$$
z(x, y)=z_{0}+\delta_{x}\left(x-x_{0}\right)+\delta_{y}\left(y-y_{0}\right)+z_{s}(x, y)
$$

where $\left(x_{0}, y_{0}, z_{0}\right)$ are the facet's center coordinates; $\delta_{x}$ and $\delta_{y}$ are the facet's slopes; and $z_{s}$ is a stochastic process whose spectrum satisfies (3.5) and (3.6).

Finally, nonlinear hydrodynamic modulation [27] must be considered. This phenomenon causes an increase of short wave height on the crests of the long wave, and a decrease of short wave height on the troughs of the long wave. Available results [7], [27] suggest that the effect of nonlinear hydrodynamic modulation can be accounted for by multiplying each short wave spectral component amplitude by $\left(1+z_{0} \kappa_{0}\right)$, where $\kappa_{0}=\left(\xi_{0}^{2}+\right.$ $\left.\eta_{0}^{2} \sin ^{2} \vartheta\right)^{1 / 2}$ is the long wave central wavenumber.

\section{Facet's Backscattering Evaluation}

In order to evaluate the reflectivity pattern, we must consider the interaction between the electromagnetic incident wave and the ocean surface. For small incidence angles, the specular reflection must be considered [9]. The dominant mechanism, for intermediate incidence angles, is widely recognized to be the Bragg resonance [2], [3], [9], which has been extended to the 
oblique incidence case [7]. For large incidence angles, shadowing and diffraction phenomena would play significant roles. However, the limiting cases of small and large incidence angles are of no practical importance whenever SAR systems are employed on ocean scenes.

The field scattered by each facet can be computed under the Kirchhoff approximation (KA); the final relation between scattered $\boldsymbol{E}^{s}$ and incident $\boldsymbol{E}^{i}$ electric fields is

$$
\left|\begin{array}{c}
E_{H}^{s} \\
E_{V}^{s}
\end{array}\right|=\frac{j k}{4 \pi R} \exp (-j k R) \boldsymbol{\gamma}\left|\begin{array}{c}
E_{H}^{i} \\
E_{V}^{i}
\end{array}\right|
$$

where $R$ is the facet center to the line of flight distance; $k=$ $\hat{\boldsymbol{k}} k$ is the incident electromagnetic wavenumber vector (lying in the plane $x=0$ ); subscripts $H$ and $V$ account for horizontal and vertical polarization components; and superscripts $i$ and $s$ account for incident and scattered fields, respectively; the matrix $\gamma$ is given by [7]

$$
\boldsymbol{\gamma}=\boldsymbol{F} D\left(\theta_{i}, \delta_{x}, \delta_{y}\right)
$$

where $\delta_{x}$ and $\delta_{y}$ are the facet's slopes along azimuth $x$ and ground range, $y$ respectively, and $\theta_{i}$ is the incidence angle. In (3.9), the matrix $\boldsymbol{F}$ accounts for the radar return polarimetric behavior due to the long wave aspect (i.e., to the facet mean plane), electromagnetic parameters dependence, and tilt modulation [7]

$$
\begin{aligned}
F_{\mathrm{HH}}= & \frac{2\left(\delta_{x} \sin \Delta \theta-\delta_{r} \cos \Delta \theta\right)}{\delta_{x}^{2}+\left(\delta_{r} \sin \Delta \theta+\delta_{s} \cos \Delta \theta\right)^{2}} \\
& \times\left[\delta_{x}^{2} R_{/ /}-\left(\delta_{r} \sin \Delta \theta+\delta_{s} \cos \Delta \theta\right)^{2} R_{\perp}\right] \\
F_{\mathrm{HV}}= & F_{\mathrm{VH}} \\
= & \frac{2 \delta_{x}\left(\delta_{s} \sin \Delta \theta-\delta_{r} \cos \Delta \theta\right)\left(\delta_{r} \sin \Delta \theta+\delta_{s} \cos \Delta \theta\right)}{\delta_{x}^{2}+\left(\delta_{r} \sin \Delta \theta+\delta_{s} \cos \Delta \theta\right)^{2}} \\
& \times\left(R_{/ /}+R_{\perp}\right) \\
F_{\mathrm{VV}}= & \frac{2\left(\delta_{s} \sin \Delta \theta-\delta_{r} \cos \Delta \theta\right)}{\delta_{x}^{2}+\left(\delta_{r} \sin \Delta \theta+\delta_{s} \cos \Delta \theta\right)^{2}} \\
& \times\left[\left(\delta_{r} \sin \Delta \theta+\delta_{s} \cos \Delta \theta\right)^{2} R_{/ /}-\delta_{x}^{2} R_{\perp}\right]
\end{aligned}
$$

where $R_{/ /}$and $R_{\perp}$ are the Fresnel reflection coefficients for parallel and perpendicular local-incident polarizations, respectively; $\Delta \theta$ is the difference between the local off-nadir angle and the one referred to the center of the footprint; $\delta_{x}, \delta_{r}$, and $\delta_{s}$ are the slopes in the radar $(x, r, s)$ coordinate system. The scalar

$$
D\left(\theta_{i}, \delta_{x}, \delta_{y}\right)=\int_{A} \exp (j 2 \mathbf{k} \cdot \boldsymbol{\rho}) d A
$$

accounts for the contribution to the scattered field due to the microstructure of the ocean surface, as detailed in the following. In (3.11), $A$ is the facet's area projected over the $(x, y)$ plane, and $\rho$ is the radial vector from the facet's center to the generic point over the facet

$$
\boldsymbol{\rho}=\left(x-x_{0}\right) \hat{\boldsymbol{x}}+\left(y-y_{0}\right) \hat{\boldsymbol{y}}+\left(z(x, y)-z_{0}\right) \hat{\boldsymbol{z}}
$$

where $z(x, y)$ is given by (3.7).

Evaluation of (3.11) is based on the observation that main contributions to the backscattered field are provided by the ripple spectral components whose wavelength is of the same order of magnitude of the incident electromagnetic wavelength. Since the height of sea waves is much smaller than their wavelength (unless breaking waves are considered), then the height of ripple spectral components involved in the scattering mechanism must be much smaller than the electromagnetic wavelength, too. Under this hypothesis, by using (3.7) and (3.12) in (3.11), expanding $\exp \left(j 2 k z_{s} \cos \theta_{i}\right)$ in series and retaining terms up to the first order, we get [2], [7]

$$
D\left(\theta_{i}, \delta_{x}, \delta_{y}\right)=j \frac{4 \pi}{\lambda} Z_{s}\left(\bar{\kappa}_{x}, \bar{\kappa}_{y}\right) \cos \theta_{i}
$$

where

$$
\left\{\begin{array}{l}
\bar{\kappa}_{x}=\frac{4 \pi}{\lambda} \delta_{x} \cos \theta_{i} \\
\bar{\kappa}_{y}=\frac{4 \pi}{\lambda}\left(\sin \theta_{i}+\delta_{y} \cos \theta_{i}\right)
\end{array}\right.
$$

and $Z_{s}(\cdot)$ is the Fourier transform of

$$
z_{s}(x, y) \operatorname{rect}\left[\left(x-x_{0}\right) / L_{x}\right] \operatorname{rect}\left[\left(y-y_{0}\right) L_{y}\right]
$$

with $L_{x} L_{y}=A$. In particular, $Z_{s}(\cdot)$ is a complex circular Gaussian random variable with mean squared value

$$
\left\langle\left|Z_{s}\left(\overline{\boldsymbol{\kappa}}_{x}, \overline{\boldsymbol{\kappa}}_{y}\right)\right|^{2}\right\rangle=A \cdot W\left(\overline{\boldsymbol{\kappa}}_{x}, \overline{\boldsymbol{\kappa}}_{y}\right)
$$

where $\langle\cdot\rangle$ stands for statistical average, and $S(\cdot)$ is the short gravity, gravity-capillary, or capillary [according to the value of the $\overline{\boldsymbol{\kappa}}=\left(\overline{\boldsymbol{\kappa}}_{x}^{2}+\overline{\boldsymbol{\kappa}}_{y}^{2}\right)^{1 / 2}$ ] wave spectrum, given by (3.5)-(3.6).

Equations (3.13) and (3.14) clearly show that, according to the Bragg phenomenon, the SAR sensor acts as a frequency selector; hence, the ocean waves that contribute to the scattering are only those resonant with the wavenumbers $\kappa$ such that (3.14) holds.

An attractive feature of (3.9) is that the matrix $\boldsymbol{F}$ accounts for the macrostructure of the problem, whereas the scalar $D\left(\theta_{i}, \delta_{x}, \delta_{y}\right)$ takes care of its microstructure. Conversely, intermediate waves affect both $\boldsymbol{F}$ and $D\left(\theta_{i}, \delta_{x}, \delta_{y}\right)$.

We finally recall that possible nonnull correlation between returns of neighboring facets with similar slopes can be accounted for [7]. This effect depends mainly on facet dimension and long wave profile.

Once the reflectivity function has been evaluated, the SAR raw signal is generated according to the procedure described in Section III-A.

From the raw signal, it is subsequently possible to get the SAR image via the usual SAR processing. As an additional feature of this simulator, focusing techniques can be tested by performing nonconventional SAR processing, as well as postprocessing feature extraction from these simulated SAR data. This is the main advantage of a SAR raw signal simulator instead of a simpler SAR image simulator: this advantage is particularly attractive for the ocean case.

\section{OIL SLICK INFLUENCE ON OCEAN SURFACE PROPERTIES}

The oil influence on the cumbersome ocean wave formation mechanism, outlined in Section II, is a very interesting issue for oceanographers. In this paper, we are concerned about the SAR-detectable side of the issue: we consider only the ocean surface effects. In this section, we discuss the ocean spectrum dependence on the presence of oil. We present a possible approach to the problem based on available models for the oil slick 
influence on the ocean surface properties. However, our simulator is modular, so that any future improvement of the model could be easily accounted for.

Oil influence on the ocean spectrum is a widely studied issue in literature, with great attention to measurements techniques [8], [31]-[39], but no sound theoretical model for the spectrum of water covered by oil is available. On the other hand, a theoretical model of ocean spectra modification in presence of surfactant is highly desirable for SAR simulation purposes. In the current literature, only empirical or semiempirical models are available, some of them being analytically related to physical modeling: these models are preferable for our scopes.

The presence of oil on the water surface reduces surface tension and friction between wind and liquid surface: the higher the oil percentage, the stronger the reduction effect [29]. This means that the wind can transfer a smaller energy to capillary waves in oil-slick-covered areas than in free-water areas. In addition, viscous dissipation increases. All this causes a decrement of the capillary spectrum intensity, which, as presented above, plays a dominant role in the scattering mechanism. Then, according to (3.13)-(3.15), a weaker electromagnetic return from areas affected by oil presence is expected, which causes darker areas in SAR images. In passim, note that surface tension reduction is a measure of sea pollution: as a matter of fact, surface tension of clean water is about $0.07[\mathrm{~N} / \mathrm{m}]$, while surface tension values lower than $0.040[\mathrm{~N} / \mathrm{m}]$ account for a pollution level that is lethal for a great diversity of marine fauna [30].

In order to quantify the above considerations, a physical approach can be followed. By using a simple energy balance equation, in equilibrium conditions we can write [8], [32], [38], [39]

$$
\begin{gathered}
\frac{d N_{w}(\boldsymbol{\kappa})}{d t}=0=Q_{w}^{\mathrm{in}}(\boldsymbol{\kappa})+Q_{w}^{\mathrm{nl}}(\boldsymbol{\kappa})-Q_{w}^{\mathrm{dis}, v}(\boldsymbol{\kappa})-Q_{w}^{\mathrm{dis}, b}(\boldsymbol{\kappa}) \\
\frac{d N_{s}(\boldsymbol{\kappa})}{d t}=0=Q_{s}^{\mathrm{in}}(\boldsymbol{\kappa})+Q_{s}^{\mathrm{nl}}(\boldsymbol{\kappa})-Q_{s}^{\mathrm{dis}, v}(\boldsymbol{\kappa})-Q_{s}^{\mathrm{dis}, b}(\boldsymbol{\kappa})
\end{gathered}
$$

wherein subscripts $w$ and $s$ refer to clean water and to oil slick, respectively. In (4.1), $N(\kappa)=(\omega / \kappa) S(\kappa)$ and $Q^{\text {in }}$ are the wind input terms; $Q^{\text {nl }}$ are the nonlinear interaction input terms; and $Q^{\text {dis, } v}$ and $Q^{\text {dis, } b}$ are the dissipation terms due to viscosity and to breaking waves, respectively. Theoretical considerations on the terms appearing in (4.1) and their analytical expressions can be found in the literature [8], [36]-[39]. A qualitative analysis of the oil influence on the source terms is also available [37]. We stress that the results reported in literature are sometimes controversial, and analytical expressions of some of the terms appearing in (4.1) (e.g., nonlinear terms and breaking-wave dissipation terms) are considered to be poorly known by all authors. In this paper, we adopt a model based on the Marangoni theory [8], [31]-[34] and on a nonlinear energy transfer theory. This model has been selected because it arises from a theoretical physical-chemical approach and provides a reasonable physical interpretation to a typical behavior of the wave damping in a range of wavenumber of great interest for SAR applications.

The terms related to breaking-wave viscous dissipation are negligible for wind speeds less than $10 \mathrm{~m} / \mathrm{s}$ [8], [32], so that we do not consider them in the following.
The wind input terms can be evaluated as [8], [39]

$$
Q_{w, s}^{\text {in }}=Q_{w, s}^{0}+\beta_{w, s} N_{w, s}
$$

i.e., as the sum of two factors. The first one, $Q_{w, s}^{0}$, is due to the resonance excitation by the atmospheric pressure pulsation, namely the Phillips' mechanism [39]. In the second one

$$
\beta_{w, s}=M \cos \varphi\left(\frac{u_{*}^{w, s}}{c_{p}}\right)^{2} \boldsymbol{\omega}
$$

where $c_{p}$ is the phase velocity of the considered wave component; $M=4 \cdot 10^{-2}$ [8]; and $\varphi$ is the angle between wave and wind directions. The frictional velocities $u_{*}$ for oil-free and oil-affected areas are [8]

$$
\begin{aligned}
u_{*}^{w} & =0.053 U_{\text {ref }} \\
u_{*}^{s} & =0.042 U_{\text {ref }}
\end{aligned}
$$

respectively, where $U_{\text {ref }}$ is a reference wind speed that can be related to the wind velocity at a $10-\mathrm{m}$ height $u_{10}$. In this paper, we use a linear relation derived from data reported in [32] and valid for wind speeds ranging from about $4 \mathrm{~m} / \mathrm{s}$ to about $10 \mathrm{~m} / \mathrm{s}$.

The viscosity dissipative terms can be expressed as [8]

$$
Q_{w, s}^{\operatorname{dis}, v}=2 c_{g} \Delta_{w, s} N_{w, s}
$$

whereas the nonlinear interaction terms are [8]

$$
Q_{w, s}^{\mathrm{nl}}=\alpha_{w, s} N_{w, s}
$$

In (4.4) and (4.5), $c_{g}$ is the wave group velocity; $\Delta_{w, s}$ is the viscous damping coefficient; and $\alpha_{w, s}$ are the nonlinear energy transfer rates. For clean water, these latter coefficients can be written as [8]

$$
\Delta_{w}=\frac{4 \kappa^{2} e \boldsymbol{\omega}}{\rho g+3 \tau \boldsymbol{\kappa}^{2}}
$$

and

$$
\boldsymbol{\alpha}_{w}=-q \boldsymbol{\beta}_{w}
$$

respectively, wherein $\rho$ is the water density; $e[\mathrm{~N} \cdot \mathrm{s} / \mathrm{m}]$ is the water dynamic viscosity [8], [32], [38]; and $q$ is a dimensionless coefficient equal to about unity, its exact value being unavailable. Simulation results (see Section VI) suggest a $q$ value equal to 1.15 .

Expressions for $\Delta$ and $\alpha$ when the oil slick is present, i.e., $\Delta_{s}$ and $\alpha_{s}$, are now in order. To explain the variations of $\Delta$ in the presence of an oil slick, Marangoni theory can be used. According to Marangoni, surfaces covered by a slick can carry two kinds of waves: the gravity-capillary and the Marangoni waves. Marangoni waves are related to surface tension gradients, caused by the oil film viscoelastic reaction to the sea surface deformations. At variance of gravity-capillary waves, Marangoni waves are longitudinal. When Marangoni and gravity-capillary waves share the same wavenumber for a given frequency, a maximum in the damping occurs. The consequence is a resonance-like behavior of the relative 
damping coefficient $l=\Delta_{s} / \Delta_{w}$. An analytical expression of this coefficient $l$ in terms of the physical-chemical properties of the slick is [8], [32], [38]

$$
l=\frac{1+X\left(\cos \theta_{L}-\sin \theta_{L}\right)+X Y-Y \sin \theta_{L}}{1+2 X\left(\cos \theta_{L}-\sin \theta_{L}\right)+2 X^{2}}
$$

where

$$
\begin{aligned}
& X=\frac{|L| \boldsymbol{\kappa}^{2}}{\sqrt{2 \boldsymbol{\omega}^{3} \rho e}} \\
& Y=\frac{|L| \boldsymbol{\kappa}}{4 \boldsymbol{\omega} e}
\end{aligned}
$$

and where $-|L| \exp \left(j \theta_{L}\right)[\mathrm{N} / \mathrm{m}]$ is the oil film complex dilational modulus, whose real and imaginary parts are related to oil elasticity and viscosity, respectively.

We come now to the factor $\alpha_{s}$. Dampening caused by Marangoni waves is not sufficient to completely explain sea surface spectrum variations in the presence of oil. As a matter of fact, according to Marangoni theory, oil slicks influence ocean waves with wavelength only lower than $1 \mathrm{~m}$, whereas experience suggests that the whole spectrum is affected by the oil presence. In addition, (4.7) is independent of wind velocity, whereas experience suggests that Marangoni damping decreases for increasing winds [8], [32], [38]. In order to quantify the whole wave-spectrum changes and wind dependence, some considerations are in order. When an external input acts on the ocean and changes its shape, the system reacts by spreading uniformly the energy through its whole spectrum via wave-wave nonlinear interactions. In other words, nonlinear interactions tend to level down peaks and fill in dips of the ocean spectrum. Hence, when an oil slick causes a strong spectrum damping around the Marangoni resonant wavelength, longer and shorter waves transfer part of their energy to the waves in the resonant spectral region, via the nonlinear wave interaction phenomenon. This is the reason why, although the damping effect directly acts only on the short waves, longer waves also are influenced by the presence of oil. Such a wave-wave interaction mechanism is strongly influenced by the wind; hence, the higher the wind intensity, the higher the energy spreading effect. And this is the reason why the (resonant) Marangoni damping decreases for increasing wind. This effect can be included by letting [8]

$$
\alpha_{s}=\alpha_{w}+\delta \alpha
$$

with

$$
\delta \alpha=\alpha_{M}\left(\frac{\kappa}{\kappa_{M}}\right)^{3 / 2}\left(\frac{u_{*}}{u_{* c}}\right)^{2} .
$$

In (4.11), $\alpha_{M}$ is the Marangoni dampening rate, equal to

$$
\alpha_{M}=2 c_{g} l\left(\kappa_{M}\right) \Delta_{w}\left(\kappa_{M}\right)=2 c_{g} \Delta_{s}\left(\kappa_{M}\right)
$$

and $\kappa_{M}$ is the Marangoni resonance wavenumber, i.e., the wave number at which the Marangoni relative damping coefficient $l$, (4.7), attains its maximum value. These constants can be calculated from Marangoni theory [8]. In addition, $u_{* c}$ is the critical frictional velocity (depending on the particular oil) at which the Marangoni damping is completely compensated for by the nonlinear energy transfer, as can be verified by using (4.12) in
TABLE I

Viscoelastic Properties of SOME OF THE SURFACTANT FluIDS COMMONLY PRESENT ON THE SEA SURFACE

\begin{tabular}{c|c|c|c|c|c|c}
\hline Substance & $\mid L /(\mathrm{N} / \mathrm{m})$ & $\theta(\mathrm{deg})$ & $f_{M}(\mathrm{~Hz})$ & $l_{\max }$ & $k_{M}\left(\mathrm{~m}^{-1}\right)$ & $\sigma_{M}\left(\mathrm{~s}^{-1}\right)$ \\
\hline Oleyl alcohol (OLA) & 0.0255 & -175 & 4.7 & 19.0 & 84.4 & 0.48 \\
\hline Acid methyl ester (PME) & 0.046 & -176 & 3.6 & 28.8 & 51.2 & 0.28 \\
\hline Oleic Acid (OLS) & 0.014 & -176 & 6.1 & 14.1 & 132.5 & 0.75 \\
\hline Hexadecanole (CEA) & 0.0315 & -175 & 4.2 & 22.9 & 68.6 & 0.39 \\
\hline Hexadecyl amine (CEAM) & 0.0290 & -172 & 4.3 & 20.4 & 71.7 & 0.38 \\
\hline Ammonium (CEAM3AB) & 0.0255 & -177 & 4.6 & 20.4 & 81.2 & 0.48 \\
\hline Tirolein (TOLG) & 0.0115 & -175 & 6.8 & 13.2 & 157.3 & 0.91 \\
\hline
\end{tabular}

(4.11), letting $\kappa=\kappa_{M}$ and $u_{*}=u_{* c}$ in (4.11) and comparing the obtained expression of $\delta \alpha$ to (4.4). This critical velocity can be theoretically evaluated in principle, but is usually experimentally determined [8].

Finally, by using (4.2a), (4.4), (4.5) in (4.1a) and (4.1b), and dividing (4.1b) by (4.1a), we get

$$
\begin{aligned}
& \frac{\left(\beta_{w}-2 c_{g} \Delta_{w}+\alpha_{w}\right) N_{w}}{\left(\beta_{s}-2 c_{g} \Delta_{s}+\alpha_{s}\right) N_{s}} \\
& \quad=\frac{\left(\beta_{w}-2 c_{g} \Delta_{w}+\alpha_{w}\right)\left(\frac{\boldsymbol{\omega} S_{w}}{\boldsymbol{\kappa}}\right)}{\left(\beta_{s}-2 c_{g} \Delta_{s}+\alpha_{s}\right)\left(\frac{\boldsymbol{\omega} S_{s}}{\boldsymbol{\kappa}}\right)}=\frac{Q_{w}^{0}}{Q_{s}^{0}} .
\end{aligned}
$$

If we assume, as it is reasonable [39], that $Q_{s}^{0}=Q_{w}^{0}$, then (4.13) provides

$$
\frac{S_{s}}{S_{w}}=\frac{\beta_{w}-2 c_{g} \Delta_{w}+\alpha_{w}}{\beta_{s}-2 c_{g} \Delta_{s}+\alpha_{s}}
$$

and can be used to evaluate the spectral damping in terms of the oil slick's physical properties.

Note that, in principle, oil slicks might also affect the spreading function $f(\kappa, \phi)$. However, it has been verified that oil slicks induce only marginal changes on this matter; we neglect this irrelevant effect and focus our attention on the $S(\kappa)$ dependence on the physical parameters of the scene and its changes whenever oil slicks are present.

The viscoelastic properties of some of the most common oils are shown in Table I. In the following, we analyze the spectrum modifications induced by the presence of oleyl alcohol (OLA) on the sea surface.

In Fig. 1, we show the damping produced by OLA presence on the ocean, evaluated according to the model presented above, at different wind intensities and with $\varphi=45^{\circ}$. According to the nonlinear wave interaction theory, the higher the wind speed, the higher the energy spreading and, hence, the weaker the Marangoni damping effect. Fig. 1 shows that the oil effect is not a simple reduction of the ocean spectrum, but an involved modification of the whole spectrum, thus making the expected image changes a more complicated issue than a simple dampening ratio (see Section $\mathrm{V}$ ). In conclusion, not only the spectral dampening but also its reshaping is crucial whenever oil is present on the water: in our ocean surface model, the whole effect is quantified and included so that the correct SAR raw signal is obtained.

\section{OIL SLICK INFLUENCE ON SAR IMAGES}

In this section, we analyze how oil slicks modify the scattering mechanism presented in Section III and their effect on the SAR image. 


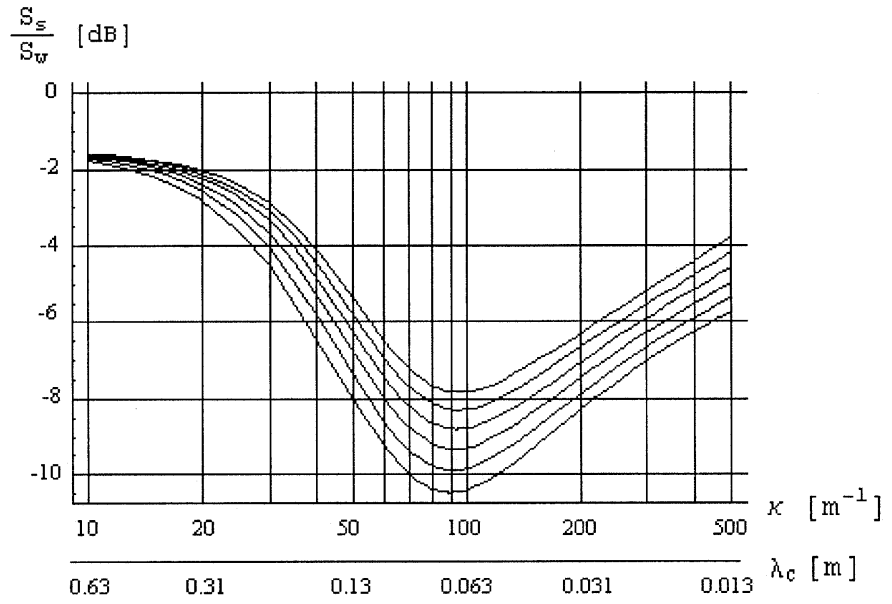

Fig. 1. Ocean spectrum damping produced by OLA, for different wind speeds, varying from $4 \mathrm{~m} / \mathrm{s}$ (lowest line) to $9 \mathrm{~m} / \mathrm{s}$ (highest line).

In order to detect oil slicks in SAR images, it is necessary that the contrast between the reflectivity values pertinent to slick-covered and free areas is appreciable. The parameter usually employed to quantify this effect is the image dampening ratio, defined as the ratio between the radar cross section of the area with and without the oil slick. This ratio depends, in an involved way, on the SAR system (frequency, polarization, look-angle, sensor velocity, pulse repetition frequency, pulse duration, chirp bandwidth) and on the ocean (directional spectra of waves), slick (concentration, surface tension, dilational modulus, and then ocean spectral dampening), and environment (wind history) parameters. A simple analytical relation between image dampening ratio and these parameters does not exist, but several studies and field campaigns [31]-[42] lead to some widely accepted conclusions. We used these studies and relevant conclusions reported below to validate our simulations (see Section VI).

The electromagnetic frequency acts as a selector on the ocean wavenumber spectrum; hence, to monitor oil presence in SAR data, the best incident wave frequencies are those corresponding to ocean waves where the spectral damping by the oil is maximum. Actual SAR images of oil slicks along with corresponding dampening ratios at different electromagnetic wavelength are available [43].

The influence of polarization is less intuitive. Several campaigns [41], [42] have assessed that image damping is always stronger for vertical-vertical (VV) rather than for horizontal-horizontal $(\mathrm{HH})$ polarization; moreover, cross polarization suffers, compared to $\mathrm{VV}$ polarization, of larger image dampening in L-band, and smaller in C-band [41].

Wind history plays a fundamental role in the appearance and detection of oil slicks. Low winds are able to generate capillary waves, but the radar echo is usually not sufficient to generate SAR images with signal-to-noise ratio high enough to allow detection of oil slicks. Conversely, high winds (speed higher than $14 \mathrm{~m} / \mathrm{s}$ [44]) tend to quickly spread the slicks so that the time interval allowed for an effective oil slick detection is reduced (an almost continuous surveying would be necessary). In addition, high winds tend to break the ocean wave structure, thus creating foam and bubbles, thus resulting in hard detection procedures.

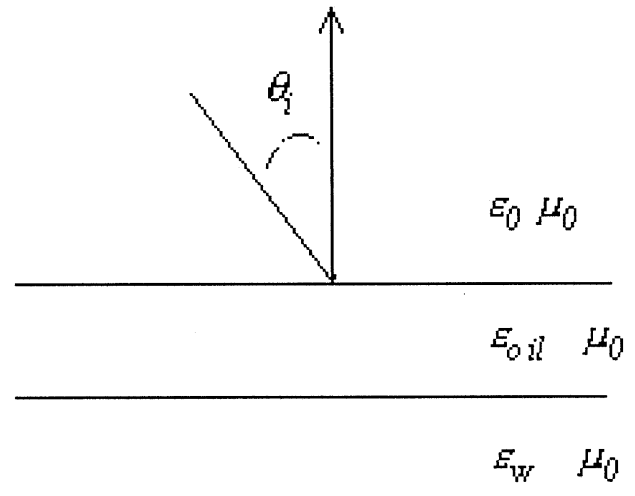

Fig. 2. Evaluation of the reflection coefficient of sea water covered by an oil layer. Geometry of the problem.

As a summary of above-cited experimental results, it can be safely stated that the most significant effect of oil slick presence on the SAR data is the dampening of the SAR image due to the dampening of capillary waves spectrum. However, other factors may play a role: in the following, we analyze in detail the influence of oil slick presence over the whole SAR raw signal formation mechanism described in Section III.

First of all, we note that the DS model, Section III-A, (3.1)-(3.3), is not influenced by the oil slick presence. In fact, the long wave dispersion equation is independent of surface tension, and long wave formation mechanism is linked to the far wind, thus being practically insensitive to the presence of a local oil slick. In other words, the macroscopic profile and its time evolution are not appreciably affected by the oil presence, and (3.1)-(3.3) still hold.

Let us now move to consider the oil layer effect on the field backscattered by each surface facet (Section III-C): typical values of the relative dielectric constant are 1.8-2.6 [2]; the oil layer thickness is usually of the order of one or fractions of a millimeter [2]. If the surface local radius of curvature is much larger than both the electromagnetic wavelength and the oil layer thickness, then the tangent plane approximation (i.e., the KA) can be used. Accordingly, surface tangential fields at each surface point can be evaluated by considering the local tangent plane and locally approximating the scattering region as a plane layered medium consisting of three regions: air, oil layer, and semiinfinite ocean (see Fig. 2). The resulting scattered field is still given by (3.8)-(3.14), with the following changes: in (3.10), the Fresnel reflection coefficients $R_{/ /}$ and $R_{\perp}$ must be replaced by the reflection coefficients $\Gamma_{/ /}$ and $\Gamma_{\perp}$ of the layered structure of Fig. 2; and in (3.13), the amplitude coefficients $Z_{s}\left(\overline{\kappa_{x}}, \overline{\boldsymbol{\kappa}_{y}}\right)$ must be modified to account for the short wave spectrum dampening. The new reflection coefficients can be easily evaluated by using, for instance, an equivalent transmission line [45]

$$
\begin{array}{r}
\Gamma_{/ /, \perp} \\
=\frac{Z_{o}\left(Z_{w}+j Z_{o} \tan \left(k_{z o} d\right)\right)-Z_{a}\left(Z_{o}+j Z_{w} \tan \left(k_{z o} d\right)\right)}{Z_{o}\left(Z_{w}+j Z_{o} \tan \left(k_{z o} d\right)\right)+Z_{a}\left(Z_{o}+j Z_{w} \tan \left(k_{z o} d\right)\right)}
\end{array}
$$

where $Z_{a, o, w}=\boldsymbol{\zeta}_{a, o, w} / \cos \boldsymbol{\theta}_{i}$ for perpendicular polarization, and $Z_{a, o, w}=\zeta_{a, o, w} \cdot \cos \boldsymbol{\theta}_{i}$ for parallel polarization; $\zeta_{a}, \zeta_{o}$, 


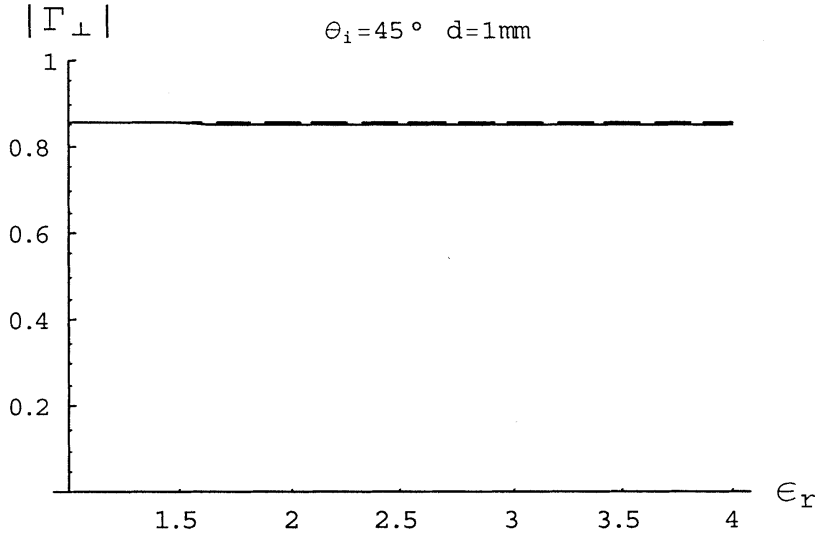

Fig. 3. Modulus of the reflection coefficient as a function of the oil relative permittivity for contaminated water (solid line) and clean water (dashed line). Perpendicular polarization $(f=5.6 \mathrm{GHz})$.

and $\zeta_{w}$ are characteristic impedance of air, oil, and water layers, respectively; and $k_{z a}, k_{z o}$, and $k_{z w}$ are the $z$ components of the wavenumber vector referring to air, oil, and water layers, respectively.

If the oil layer thickness is much smaller than the wavelength, then we again obtain the air-water interface Fresnel reflection coefficients, and the effect of the presence of oil on the reflection coefficients is negligible. In order to verify if this is the case for oil layer thickness and electromagnetic wavelength values of practical interest, some numerical examples are reported.

We consider a 1-mm oil layer (typical value for actual cases [2]), a plane wave with frequency $f=5.6 \mathrm{GHz}$ (a different choice in the microwave range would not lead to significant differences in the results), a local incident angle of $\theta_{i}=45^{\circ}$ (typical average value for actual SAR systems), and the dielectric constant of water $\epsilon_{w}=80+4 j$ [2] (typical value for the ocean environment at typical SAR system frequencies). Both perpendicular and parallel polarizations are analyzed.

In Fig. 3, $\left|\Gamma_{\perp}\right|$ as a function of the oil relative permittivity $\epsilon_{\mathrm{oil}}$ is shown for both contaminated (continuous line) and clean water (dashed line) in the $\epsilon_{\text {oil }}$ range 1-4, which includes typical values for actual oils (1.8-2.6). This figure leads to argue that the influence of the typical oil thickness on the reflection coefficient is extremely weak. The same analysis has been performed for parallel polarization. The dependence of the reflection coefficient on the oil relative permittivity $\epsilon_{\text {oil }}$ is almost the same as the previous one; thus, we can reach analogous conclusions.

The weak effect of the oil permittivity is related mainly to the low layer thickness with respect to the electromagnetic wavelength. Such a condition, at microwave frequencies, is always verified if natural oil is present on water [2]. In most of the cases in which artificial oil has been discharged in water, the oil layer thickness is still lower than a few millimeters. In rare cases of particularly intensive emission (e.g., a tank disaster), the oil layer thickness can reach higher values. Thus, it is important to test how the reflection coefficient changes in such cases.

Let the oil permittivity be set to the value $\epsilon_{\text {oil }}=2.5$. In Fig. 4, $\left|\Gamma_{\perp}\right|$ and $\left|\Gamma_{/ /}\right|$as a function of the oil layer thickness $d$ is shown for both contaminated and clean water. This figure shows that for increasing layer thickness, the value of $\left|\Gamma_{\perp}\right|$ begins to significantly change.

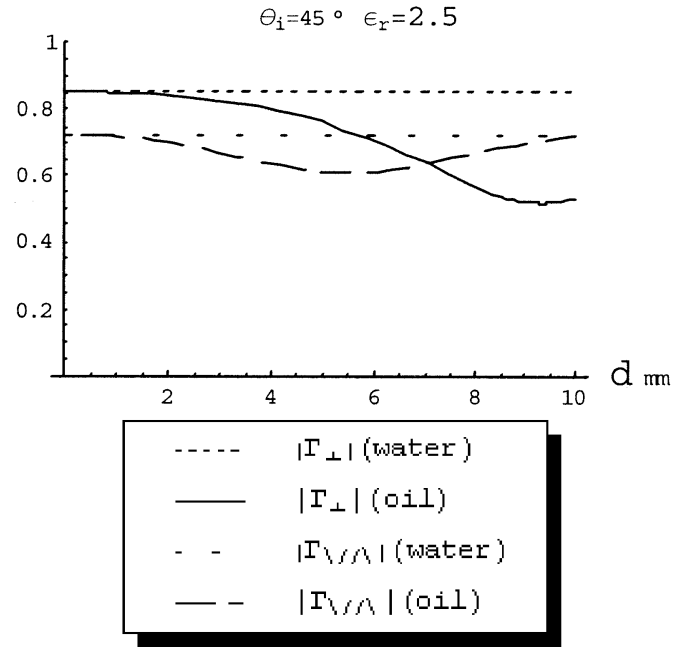

Fig. 4. Modulus of the reflection coefficient as a function of the oil layer thickness $d$ for contaminated water and clean water and for both perpendicular and parallel polarizations $(f=5.6 \mathrm{GHz})$.

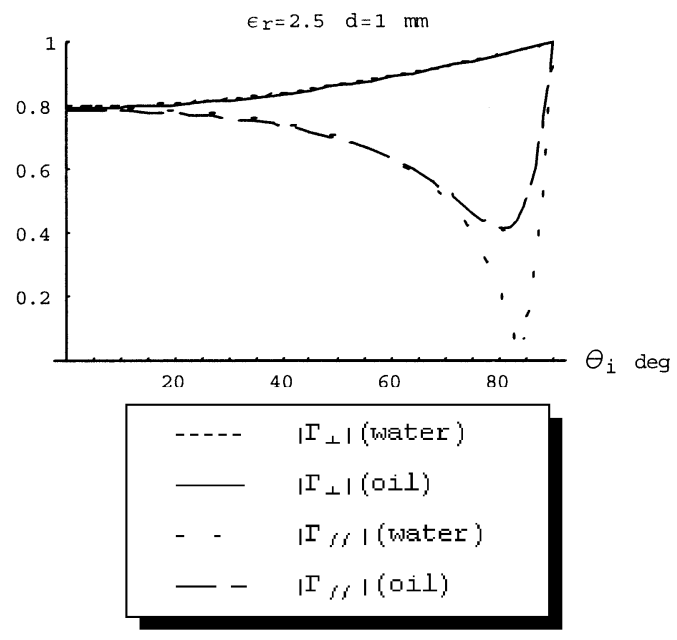

Fig. 5. Modulus of the reflection coefficient as a function of the incidence angle for contaminated water and clean water and for both perpendicular and parallel polarizations $(f=5.6 \mathrm{GHz})$.

The oil effect begins to be appreciable as $d$ is increased also on $|\Gamma / /|$, but the change is weaker compared to the perpendicular polarization.

It is meaningful to explore the behavior of reflection coefficients as a function of the local incidence angle, hence of the scattering area location on the long wave. In Fig. 5, we show that the oil presence does not change $\left|\Gamma_{\perp}\right|$, while in $\left|\Gamma_{/ /}\right|$behavior we recognize that a pseudo-Brewster phenomenon arises if the water is not covered by the oil layer. The presence of oil leads to miss the Brewster angle, and this significantly changes the reflection coefficient. But it is important to outline that the most significant variations are relevant to a range of incidence angles $\left(70^{\circ}-90^{\circ}\right)$ that are not typical of actual cases. For actual incidence angles values, the changes of $\left|\Gamma_{/ /}\right|$are slightly appreciable.

The above analysis shows that the oil permittivity weakly influences the electromagnetic return in most of the actual cases; this result becomes evident if the oil layer thickness is limited to be less than few millimeters. However, our simulator can easily 
include this effect by using the expression of the reflection coefficients $\Gamma_{/ /}$and $\Gamma_{\perp}$ of the layered structure of Fig. 2.

Note also that the Bragg resonant wavelengths [see (3.14)] are not changed by the oil's presence. As a matter of fact, they depend on the facet's slopes and, hence, on the long wave profile that as already stated is practically not affected by the presence of oil. Thus, considerations on the scattering mechanism reported in Section III also apply to the case of oil presence. Similarly, the correlation between returns of neighboring facets (see Section III-C) is not significantly affected by the presence of oil, since it depends on the facet's dimensions and on the long wave profile. Finally, also the nonlinear hydrodynamic modulation, at least in our framework (see Section III-C) is not influenced by the oil slick, but for the capillary wave spectrum modification reported in Section IV.

As a conclusion, the gravity-capillary waves spectral damping is usually the dominant phenomenon in the evaluation of changes occurring to the radar cross section whenever oil is present on the ocean surface. This phenomenon is included in the proposed simulation procedure by modifying (3.15): when an oil slick is present, the amplitude coefficients must be changed by multiplying the spectrum of (3.5) and (3.6) by the spectral damping ratio (4.14). However, other physical effects that have been shown to be of marginal relevance can be easily included in the simulator for precise generation of simulated SAR raw data relevant to oil slicks.

\section{Simulation EXPERIMENTS}

In this section, we present and discuss some meaningful experiments performed by implementing the model presented in previous sections in order to show potentialities of the simulator. In addition, we present an innovative analysis of the images generated from the simulated SAR raw signal, suggesting new tools to study oil slicks on the ocean surface.

In the experiments listed below, we consider an ocean swell with central wavenumber $0.027 \mathrm{~m}^{-1}$ (i.e., wavelength equal to $232.7 \mathrm{~m}$ ) and a bandwidth of $0.01 \mathrm{~m}^{-1}$ [7]. The swell travels along a direction tilted $45^{\circ}$ with respect to the azimuth. On such a structure, a spectrum of short waves is superimposed whose characteristics are determined by (3.5) and (3.6) and by a spreading function $f(\kappa, \phi)=\cos ^{2}\left(\phi-\phi_{0}\right), \phi_{0}$ being the direction of the local wind. The scene is divided in two regions: the upper part is affected by surfactant materials, while the lower is clean water.

We consider the SIR-C sensor, working in L-, C-, and X-band operation mode. Relevant sensor parameters, for the considered frequency bands, are given in Table II. We simulated the SAR raw signal; to show the effects on SAR images, a standard processing has been performed. In particular, no focus adjustment has been considered [46]. Resolution of all the simulated images is of $17.6 \mathrm{~m} \times 18.32 \mathrm{~m}$ obtained by means of a spatial multilook averaging four azimuth pixels, in order to get an almost square pixel for visual purposes. All the other results that we present refer to single-look images. We performed different experiments by varying wind speed and direction, electromagnetic frequency, and the kind of oil.
TABLE II

MAIN SIR-C SYSTEM PARAMETERS

\begin{tabular}{c|c|c|c}
\hline & L Band & C Band & X Band \\
\hline Frequency [GHz] & 1.254 & 5.3 & 9.61 \\
\hline Polarization & VV & VV & VV \\
\hline PRF [Hz] & 1736 & 1736 & 1736 \\
\hline Platform Velocity [Km/s] & 7.7 & 7.7 & 7.7 \\
\hline Platform Altitude [Km] & 225 & 225 & 225 \\
\hline Look Angle [deg] & 46.7 & 46.7 & 46.7 \\
\hline Azimuth resolution [m] & 4.44 & 4.44 & 4.44 \\
\hline Range resolution [m] & 18.32 & 18.32 & 18.32 \\
\hline
\end{tabular}

In the first set of simulations, the considered substance is OLA, which is one of the most commonly employed oils in experimental studies [8], [32], [38] and whose effects on the ocean spectrum have been presented in Section IV. In addition, wind direction forms a $45^{\circ}$ angle with the wave direction of propagation.

We consider first simulation in the L-band. The SAR carrier frequency is equal to $f=1.25 \mathrm{GHz}$; thus, the resonant wavelengths are around $30 \mathrm{~cm}\left(\kappa\right.$ is about $\left.21 \mathrm{~m}^{-1}\right)$, i.e., in a range heavily influenced by the gravity strength. Fig. 6(a)-(c) refers to wind intensities of 5,7, and $9 \mathrm{~m} / \mathrm{s}$ measured at $10 \mathrm{~m}$ above the surface. We notice that the oil-covered zone is darker than the clean one. The contrast between the two areas decreases as the wind speed increases. This confirms theoretical derivation that detection capability depends on the wind speed and that the Marangoni damping decreases for increasing winds (see Section IV). A quantitative analysis of the presented images has been performed by evaluating the image damping ratios. In the upper part of Table III, the obtained results are presented. Such a table provides a confirmation of the qualitative considerations obtained by a visual inspection of the images.

Let us now move to the C-band. In Fig. 7(a)-(c), the wind speed is 5,7 , and $9 \mathrm{~m} / \mathrm{s}$, respectively: again, we note how the contrast becomes weaker as the wind speed is increased. Table III still provides a quantitative confirmation of the above-presented qualitative considerations. It is also interesting to note that, at the same wind speed, damping at C-band is stronger than at L-band (e.g., see Figs. 9(a) and 10(a) and corresponding values in Table III). This is easily explained by considering that, at $\mathrm{C}$-band, the resonant wavelengths are on the order of $5 \mathrm{~cm}\left(\kappa\right.$ on the order of $\left.125 \mathrm{~m}^{-1}\right)$ and that the surface spectra are more damped than at L-band-resonant wavelengths (see Fig. 1).

For the X-band sensor, the simulated images are shown in Fig. 8(a)-(c), with the wind speed of 5, 7, and $9 \mathrm{~m} / \mathrm{s}$, respectively. Again, we note how the contrast becomes weaker for higher wind speeds. In Table III, we show the obtained damping ratios. By comparing Figs. 7(a) and 8(a) and the corresponding damping values in Table III, we note that, at the same wind speed, the damping effect is slightly stronger at $\mathrm{C}$ - than at X-band. This is consistent with the fact that, at C-band, the surface-resonant wavelength spectrum damping is a little stronger in correspondence with $\mathrm{C}$-band than at $\mathrm{X}$-band-resonant wavelengths $(\lambda=3 \mathrm{~cm}$; hence, $\kappa$ is on the order of $210 \mathrm{~m}^{-1}$; see Fig. 1). 


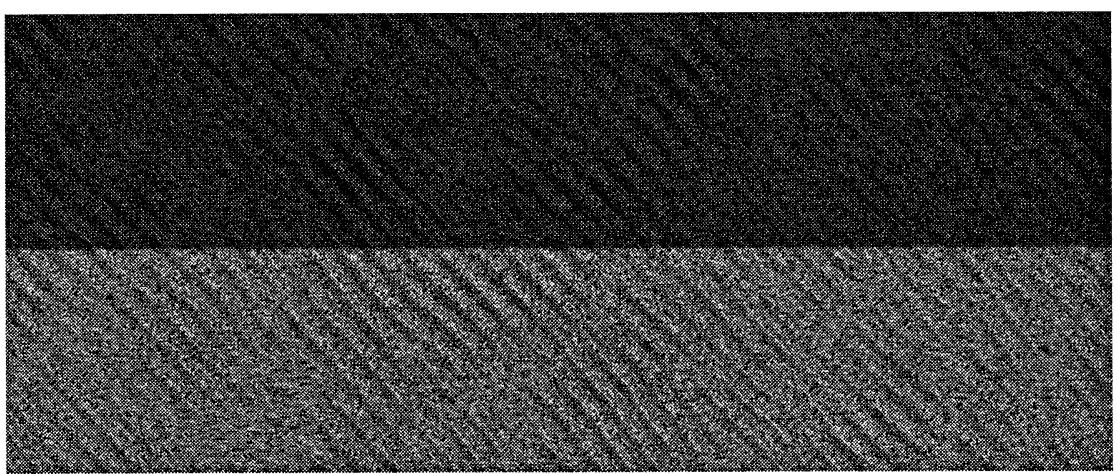

(a)

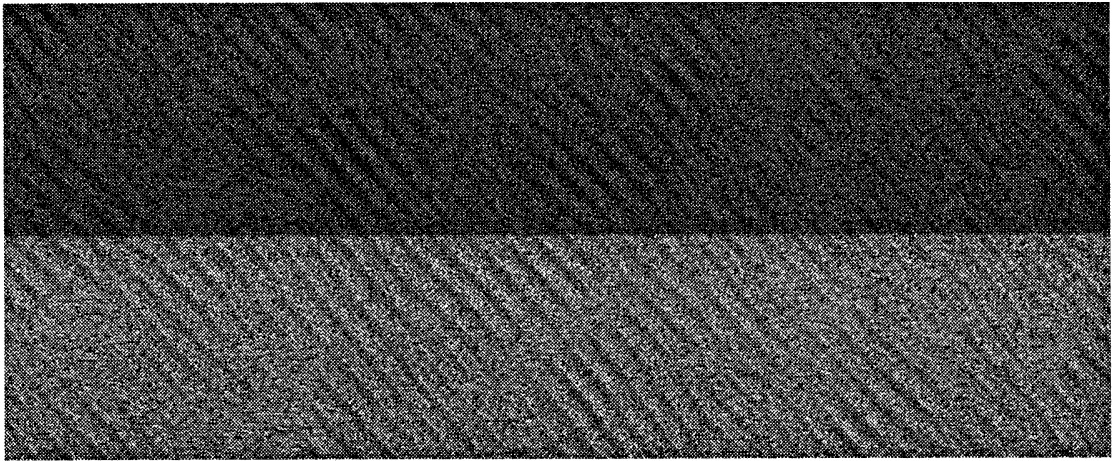

(b)

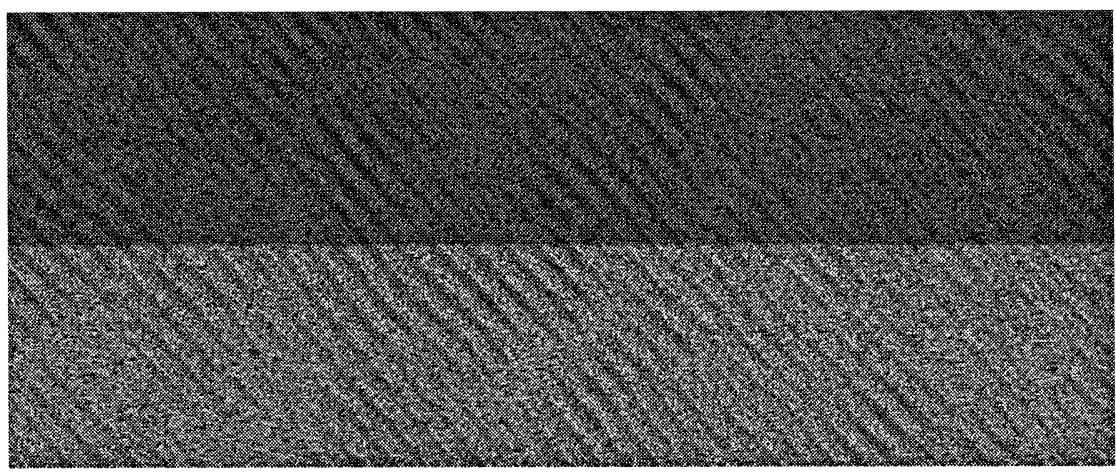

(c)

Fig. 6. Simulation of the scene sensed by the SIR-C sensor in L-band for (a) $u_{10}=5 \mathrm{~m} / \mathrm{s}$; (b) $u_{10}=7 \mathrm{~m} / \mathrm{s}$; and (c) $u_{10}=9 \mathrm{~m} / \mathrm{s}$. The upper region of the picture is sea covered by OLA, while the lower is clean sea water.

TABLE III

DAMPING Ratios PRODUCED By OLA, EVALUATEd FROM THE SAR SIMULATED IMAGES

\begin{tabular}{c|c|c|c|c}
\hline SENSOR & WIND SPEED $(\boldsymbol{m} / \mathbf{s})$ & SUBSTANCE & Wind Direction & DAMPING (dB) \\
\hline SIR-C L & & & & \\
\hline & 5 & OLA & $45^{\circ}$ & -4.12 \\
\cline { 2 - 5 } & 7 & OLA & $45^{\circ}$ & -3.90 \\
\cline { 2 - 5 } & 9 & OLA & $45^{\circ}$ & -3.61 \\
\hline SIR-C C & & & & \\
\hline \multirow{4}{*}{} & 5 & OLA & $45^{\circ}$ & -7.20 \\
\cline { 2 - 5 } & 7 & OLA & $45^{\circ}$ & -6.61 \\
\cline { 2 - 5 } & 9 & OLA & $45^{\circ}$ & -5.91 \\
\cline { 2 - 5 } & 7 & OLA & $30^{\circ}$ & -6.26 \\
\hline X SAR & 7 & OLA & $70^{\circ}$ & -7.66 \\
\hline & & & & -5.88 \\
\cline { 2 - 5 } & 7 & OLA & $45^{\circ}$ & -5.03 \\
\cline { 2 - 5 } & 9 & OLA & $45^{\circ}$ & -4.45 \\
\hline
\end{tabular}

The above results, along with other ones obtained for different wind directions, are summarized in Fig. 9 and are compared with experimental results collected during the SIR-C/X-SAR mission and available in the literature [38]. In particular, in Fig. 9 the sets of image-damping values measured over SIR-C images of OLA slicks under wind conditions comparable with those used in our simulations are represented by the vertical segments, while simulated SAR image-damping values are represented by symbols described at the right side of the figure. It must be noted that (with the exception of wind velocity) environmental conditions corresponding with actual SAR acquisitions are not available to us, so that they may not match those used in our simulations. In spite of that, Fig. 9 shows that the performed simulations are in excellent agreement with the experimental results. In particular, in both actual and simulated data, the stronger damping is obtained at $\mathrm{C}$ - and $\mathrm{X}$-bands. This provides a strong confirmation of the validity of the simulator and of the employed models. 


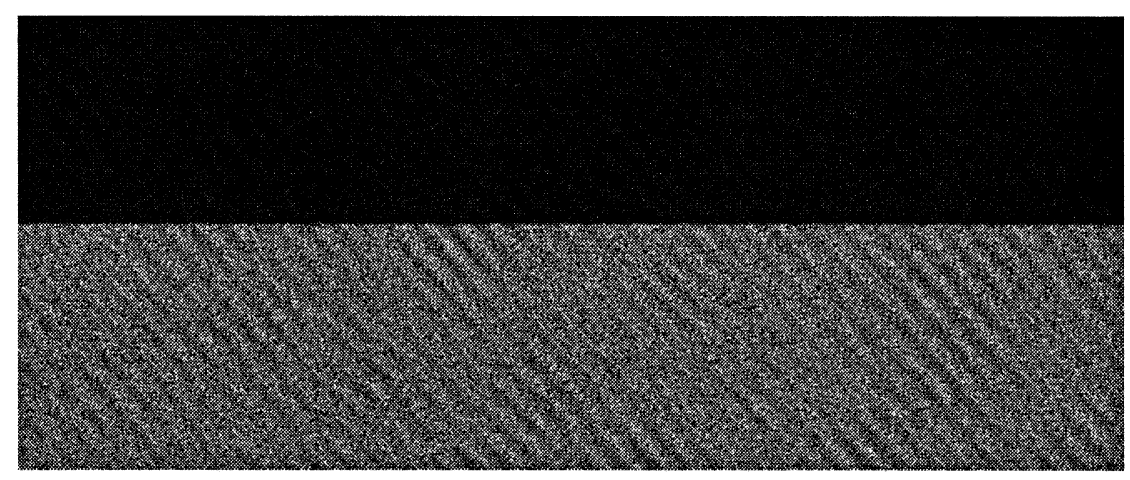

(a)

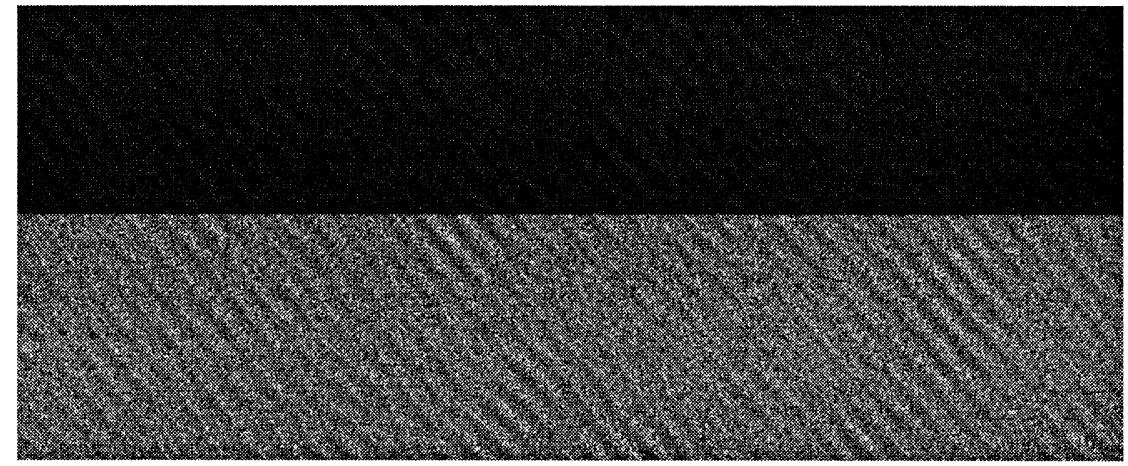

(b)

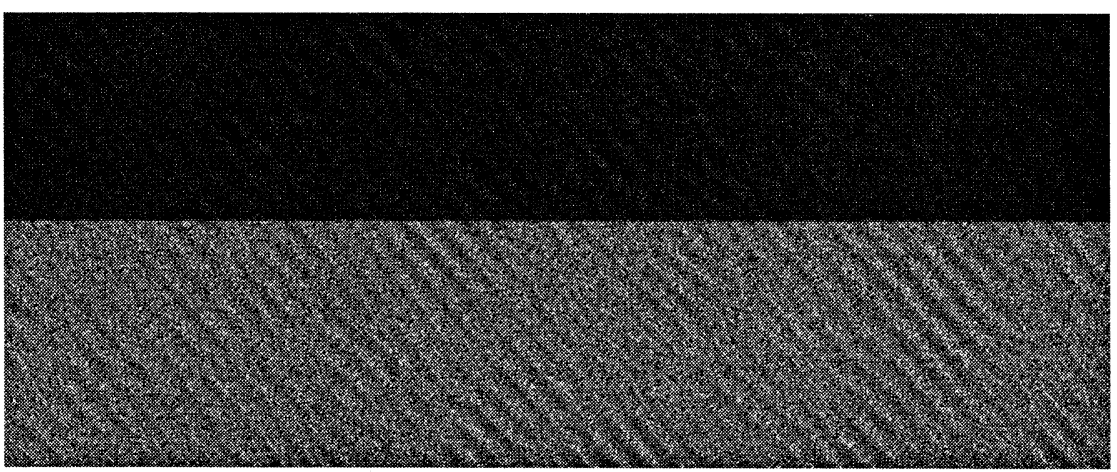

(c)

Fig. 7. Simulation of the scene sensed by the SIR-C sensor in C-band for (a) $u_{10}=5 \mathrm{~m} / \mathrm{s} ;$ (b) $u_{10}=7 \mathrm{~m} / \mathrm{s} ;$ (c) $u_{10}=9 \mathrm{~m} / \mathrm{s}$. The upper region of the picture is sea covered by OLA, while the lower is clean sea water.

Then, we performed some simulations by considering different substances. For instance, results corresponding to a palmitic acid methyl ester (PME), whose viscoelastic behavior is similar to the one of natural biogenic oil films [38], are collected in Table IV. In this case, the stronger damping is obtained at L-band. This behavior is in agreement with experimental results over natural slicks [38].

The image damping, well simulated by the presented simulator, is a well-known effect related to the reduction of the sea spectrum in the sensor wavenumber range. In the following, we perform an innovative analysis, by evaluating the normalized intensity moments of the obtained images, defined as

$$
\mathrm{NIM}=\frac{I_{n}}{\left(I_{1}\right)^{n}} \text { where } I_{n}=\int x^{n} f(x) d x
$$

and $f(x)$ is the probability density function of $x$.
Normalized intensity moments provide information on the pixel intensity distribution around the mean value. In Fig. 10, we show the normalized intensity moments evaluated from the L-band SAR image shown in Fig. 6(a). In an analogous way, normalized intensity moments are evaluated for $\mathrm{C}$ - and $\mathrm{X}$-band images, and the corresponding values are shown in Figs. 11 and 12 , respectively. Obtained results show that the higher order normalized intensity moments of the clean water may differ by about one order of magnitude from the corresponding normalized intensity moments of oil-affected water. This result offers a new technique to detect an oil slick on the ocean surface.

In order to test the possibility of employing the presented method to reduce ambiguity causes, we performed a simulation of an ocean scene analogous to that of Figs. 6-8 in which the oil-covered region is replaced by a clean water region with a lower wind, such that the resulting damping is the same of that 


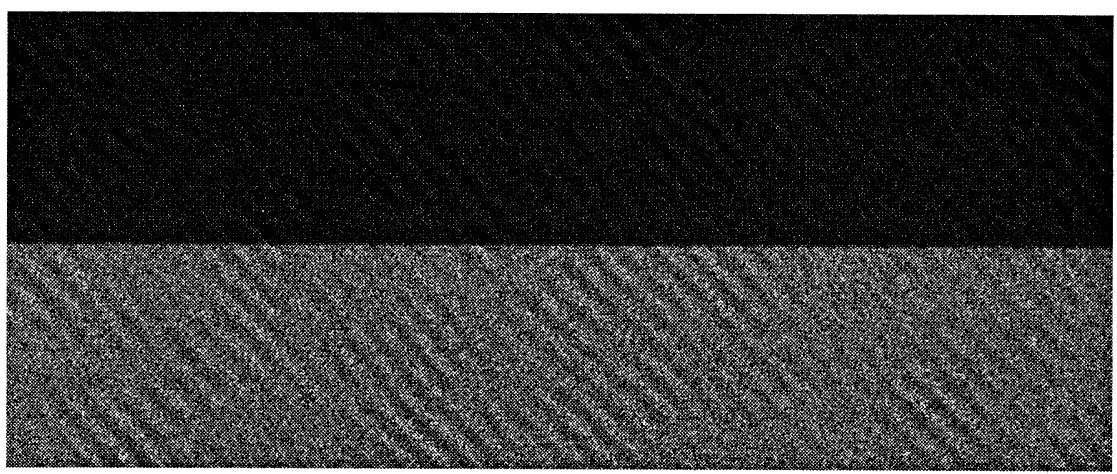

(a)

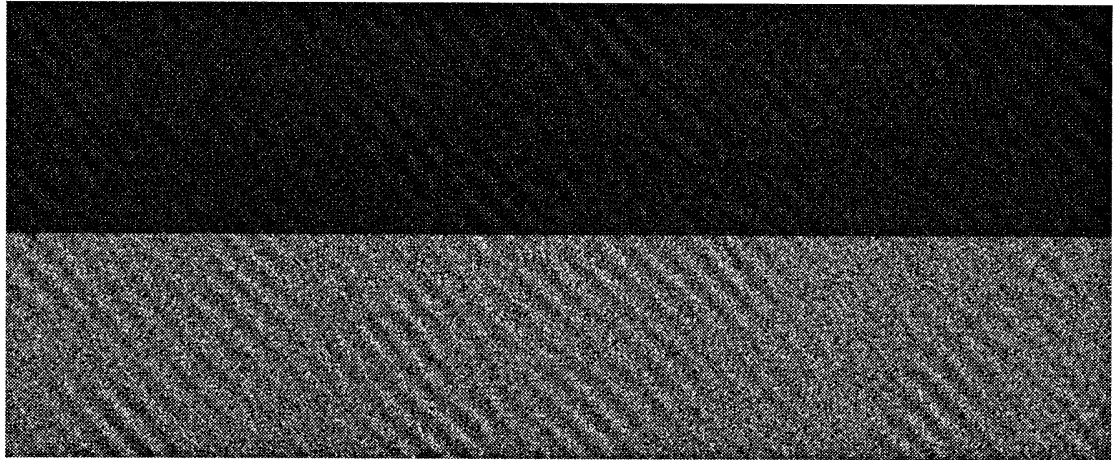

(b)

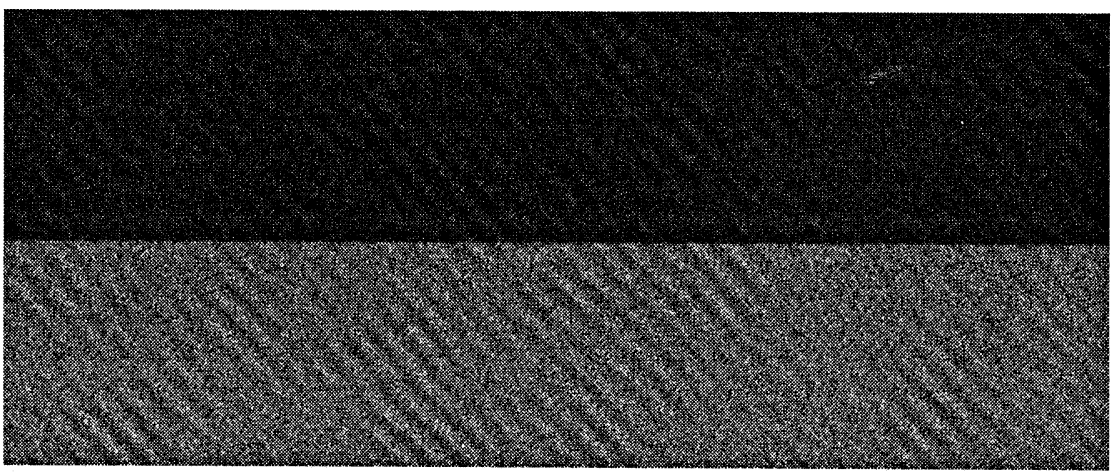

(c)

Fig. 8. Simulation of the scene sensed by the SIR-C sensor in X-band for (a) $u_{10}=5 \mathrm{~m} / \mathrm{s}$; (b) $u_{10}=7 \mathrm{~m} / \mathrm{s}$; (c) $u_{10}=9 \mathrm{~m} / \mathrm{s}$. The upper region of the picture is sea covered by OLA, while the lower is clean sea water.

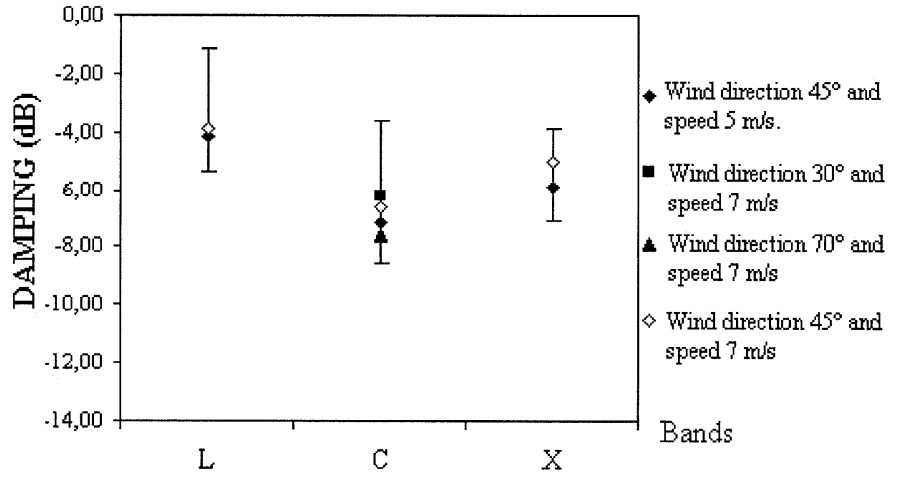

Fig. 9. Comparison between image-damping ratios measured over simulated and actual images of OLA slicks at L-, C-, and X-bands. The ranges of values measured over SIR-C actual data, for wind speeds from $4-7 \mathrm{~m} / \mathrm{s}$, are represented by vertical segments, whereas values measured over simulated SAR data are represented by symbols. generated in Figs. 6-8. The normalized intensity moments have been then evaluated. Obtained results (see Fig. 13) show that the lack of wind, at variance with the presence of an oil slick, does not significantly change the normalized intensity moments. This analysis may offer a new technique to discriminate, in SAR imagery, dark areas caused by oil slicks from those caused by lack of wind. Such a technique should be applied to dark areas large enough to perform a reliable moment estimation.

An interpretation of this different behavior of normalized intensity moments is now in order. The Bragg resonant wavenumber, hence the value of the Bragg resonant spectral component amplitude, slightly changes from pixel to pixel around a mean value, due to the varying mean plane slope, in turn related to long and intermediate waves. This causes a variation of the pixel radar return around a mean value [see (3.15)]. The distribution of radar returns around their mean value is, therefore, related to the shape of the sea spectrum 
TABLE IV

Damping Ratios PRoduced by PME, Evaluated FROM THE SAR SIMULATED IMAGES

\begin{tabular}{c|c|c|c|c}
\hline SENSOR & WIND SPEED $(\boldsymbol{m} / \boldsymbol{s})$ & SUBSTANCE & Wind Direction & DAMPING (dB) \\
\hline SIR-C L & 5 & PME & $45^{\circ}$ & -11.66 \\
\hline & 7 & PME & $45^{\circ}$ & -9.02 \\
\cline { 2 - 5 } & 9 & PME & $45^{\circ}$ & -7.09 \\
\hline \multirow{2}{*}{ SIR-C C } & 5 & PME & $45^{\circ}$ & -6.77 \\
\hline & 7 & PME & $45^{\circ}$ & -6.10 \\
\cline { 2 - 5 } & 9 & PME & $45^{\circ}$ & -5.49 \\
\hline X SAR & 5 & PME & $45^{\circ}$ & -5.36 \\
\hline & 7 & PME & $45^{\circ}$ & -4.83 \\
\hline & 9 & PME & $45^{\circ}$ & $-4,36$ \\
\hline
\end{tabular}

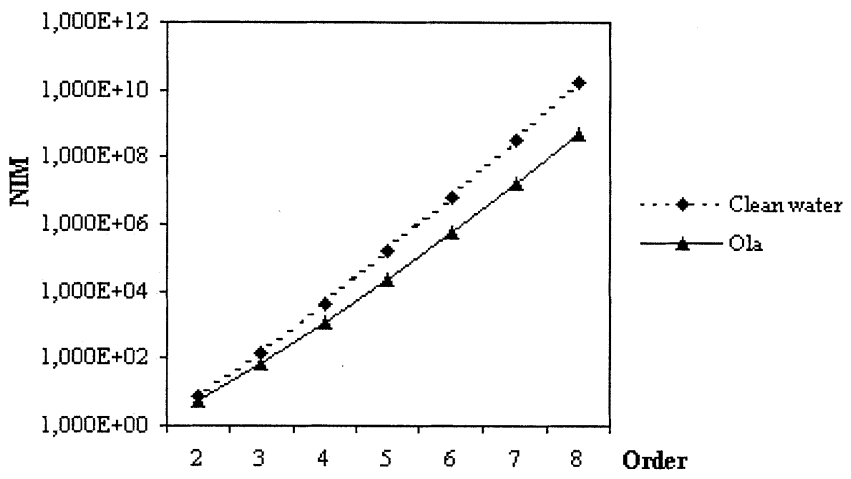

Fig. 10. Normalized intensity moments evaluated on the simulated L-band image of Fig. 6 at a wind speed of $u_{10}=5 \mathrm{~m} / \mathrm{s}$. Similar behavior is observed at higher wind speeds.

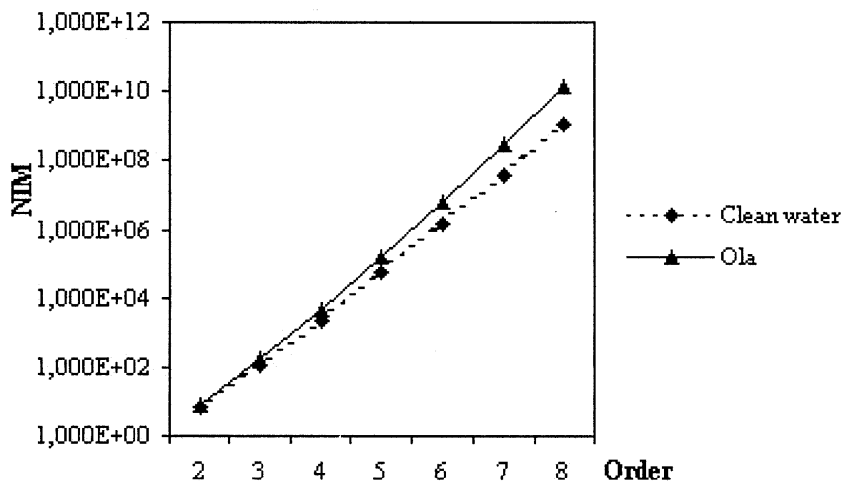

Fig. 11. Normalized intensity moments evaluated on the simulated C-band image of Fig. 7 at a wind speed of $u_{10}=5 \mathrm{~m} / \mathrm{s}$. Similar behavior is observed at higher wind speeds.

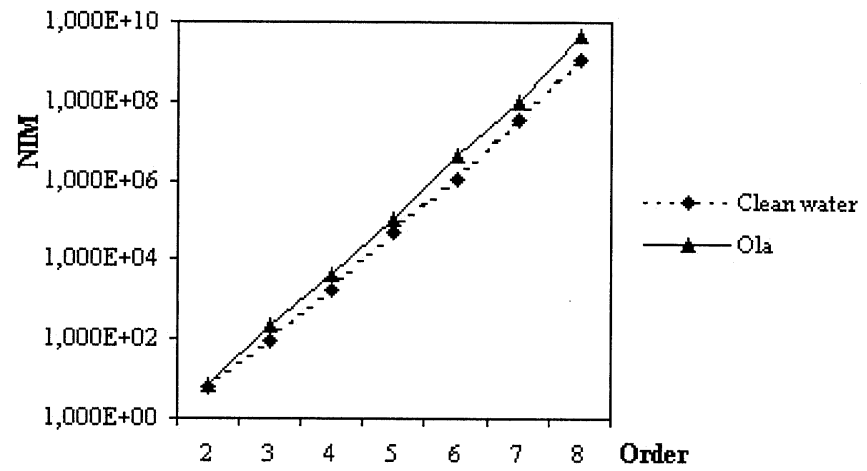

Fig. 12. Normalized intensity moments evaluated on the simulated X-band image of Fig. 8 at a wind speed of $u_{10}=5 \mathrm{~m} / \mathrm{s}$. Similar behavior is observed at higher wind speeds.

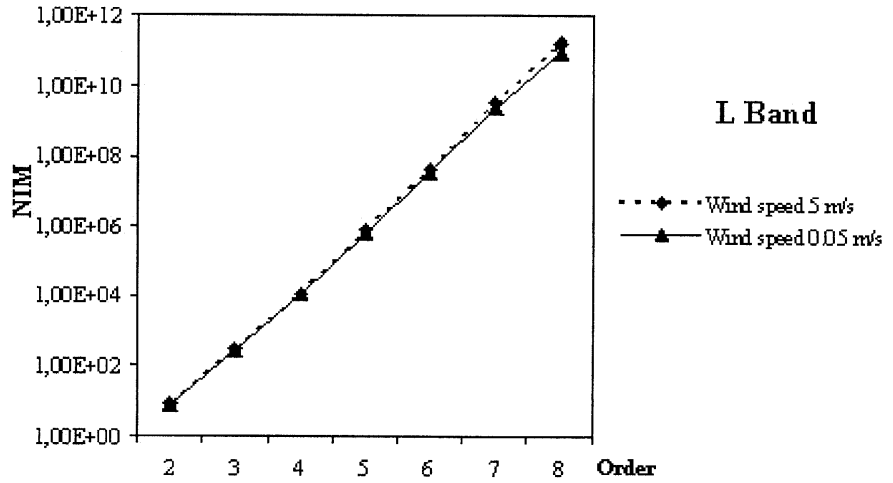

Fig. 13. Normalized intensity moments evaluated on a simulated L-band image of an ocean scene analogous to that of Fig. 6, in which the oil-covered region is replaced by a clean-water region with a lower wind, such that the resulting damping is the same as that generated in Fig. 6.

around the mean Bragg wavenumber. While a decrease of wind velocity does not substantially changes the sea spectrum shape, and only reduces its whole amplitude, the presence of an oil slick substantially changes the sea spectrum shape, especially in correspondence with the Marangoni spectrum dip (see Section IV); in addition, at microwave frequencies, the Bragg resonant wavenumber is usually placed just in correspondence with the Marangoni spectrum dip. This change of the sea spectrum shape in the presence of an oil slick explains the measured statistic normalized moment change in SAR images of oil-covered sea.

\section{CONCLUSION}

Oil slicks detection with spaceborne sensors is a goal of great relevance for its theoretical and applicative implications. The presence of oil in water causes a dynamic combination of physical phenomena that are issues of controversial oceanographic studies. Wind history, water temperature, age and concentration of the slick, its properties of interacting with sea and atmosphere, and its dielectric and geometric signatures must be carefully accounted for. In this paper, we presented a SAR raw signal simulator, built to explore potentialities of the radar instruments to detect the presence of oil on the sea and to provide a potential tool to correctly read available data.

A SAR raw signal simulator is a powerful tool for the creation of algorithms for oil slick detection. Besides, a good simulation is an important step if we are interested in identifying the nature of the slick. Use of neural networks [47] or fractal dimension study of the slick [48] are going to be employed to detect the shape of the slick, hence its nature (artificial slicks have more regular shape than natural slicks). Our approach is complementary to those methods: we focused our attention on the electromagnetic mechanisms that contribute to the SAR signal formation, paying attention to the oil slick influence on them. This allowed to quantitatively evaluate the effect of an oil slick on both mean value and higher order statistics of the SAR image intensity.

We described the ocean surface by means of a multiscale model, with a swell on which a continuous spectrum of short waves is superimposed. Intermediate waves are accounted for by means of a tilting effect. The scene is divided in planar 
facets from which the electromagnetic return is evaluated under Kirchhoff and physical-optics approximation. The effects of the ocean waves movements and the orbital speed of the water particles are accounted for by means of the DS model and the nonlinear hydrodynamic modulation theory, respectively.

The oil influence on the sea surface spectrum has been included by using a model based on Marangoni theory and a nonlinear energy transfer theory. The influence of the oil dielectric constant over the scattered field has been analyzed as well, and it was recognized to be usually negligible (except that under very particular conditions), because the oil layer width is a small fraction of the electromagnetic wavelength. However, this effect is included in the simulator.

Finally, some meaningful simulations have been presented. Oil slick image damping measured on obtained simulated SAR images turns out to be in good agreement with those reported in literature and measured over real SAR images for the same oil and comparable wind conditions. For instance, they confirm that damping is stronger at C- and X-bands for OLA oil slicks, whereas the damping is stronger at L-band for PME oil slicks. Due to the cumbersome nature of the physical mechanism involved in the problem and to the highly nonlinear behavior of the whole SAR raw signal simulation procedure, these results are certainly nontrivial. Besides, simulations were used to introduce an innovative analysis of the SAR images, suggested by studying the normalized moments of intensity of simulated images. Obtained results suggest the normalized intensity moment as a possible new tool to discriminate, in SAR images, dark areas caused by oil slicks from those caused by lack of wind.

\section{REFERENCES}

[1] R. P. La Belle and C. M. Anderson, "Offshore oil-spill occurrence rates," in Proc. OCEANS'96, vol. 2, 1996, pp. 751-753.

[2] F. T. Ulaby, R. K. Moore, and A. K. Fung, Microwave Remote Sensing: Active and Passive. Reading, MA: Addison-Wesley, 1982.

[3] I. S. Robinson, Satellite Oceanography. New York: Wiley, 1985.

[4] J. C. Scott, "Ocean surface slicks - Pollution, productivity, climate and life saving," in Proc. IGARSS, Hamburg, Germany, p. 1463.

[5] E. A. Ermakov, I. A. Sergievskaya, E. H. Zuikova, V. Y. Goldbet, and J. C. Scott, "Field studies of natural slicks due to internal waves," in Proc. IGARSS, Hamburg, Germany, pp. 167-9.

[6] J. Lu, H. Lim, S. C. Liew, M. Bao, and L. K. Kwoh, "Ocean oil pollution mapping with ERS SAR imagery," in Proc. IGARSS, Hamburg, Germany, p. 212

[7] G. Franceschetti, M. Migliaccio, and D. Riccio, "On ocean SAR raw signal simulation," IEEE Trans. Geosci. Remote Sensing, vol. 36, pp. 84-100, Jan. 1998.

[8] W. Alpers and H. Huhnerfuss, "The damping of ocean waves by surface films: A new look at an old problem," J. Geophys. Res., vol. 94, pp. $6251-6265,1989$

[9] G. R. Valenzuela, "Theories for the interaction of electromagnetic and ocean waves-A review," Bound.-Layer Meteorol., vol. 13, pp. 61-85, 1978.

[10] R. O. Harger, "SAR ocean imaging mechanisms," in Spaceborne Synthetic Aperture Radar for Oceanography, R. C, Beal, P. S. DeLeonibus, and I. Katz, Eds. Baltimore, MD: John Hopkins Univ. Press, 1981, pp. $41-52$.

[11] R. O. Harger and C. E. Korman, "Comparisons of simulated and actual synthetic aperture radar gravity wave images," J. Geophys. Res., vol. 93, pp. $13867-13882,1988$

[12] R. O. Harger, "The synthetic aperture radar image of time-variant scenes," Radio Sci., vol. 15, pp. 749-756, 1980

[13] R. K. Raney and P. W. Vachon, "Synthetic aperture radar imaging of ocean waves from an airborne platform: Focus and tracking issues," $J$. Geophys. Res., vol. 93, pp. 12457-12486, 1988.
[14] J. R. Apel, Principles of Ocean Physics. San Diego, CA: Academic, 1988.

[15] B. Kinsman, Wind Waves. Mineola, NY: Dover, 1983.

[16] G. Neumann and W.J. Pierson, Principles of Physical Oceanography. Englewood Cliffs, NJ: Prentice-Hall, 1996.

[17] A. Defant, Physical Oceanography. New York: Pergamon, 1961, vol. I-II.

[18] R. E. Glazman and S. H. Pilorz, "Effects of sea maturity on satellite altimeter measurements," J. Geophys. Res., vol. 95, no. C3, pp. 2857-2870, 1990.

[19] D. P. Kasilingam and O. H. Shemdin, "The validity of the composite surface model and its application to the modulation of the radar backscatter," Int. J. Remote Sens., vol. 13, pp. 2079-2104, 1992.

[20] G. Franceschetti, M. Migliaccio, and D. Riccio, "Concepts for an efficient SAR ocean simulator," in Proc. IGARSS, pp. 1328-1330.

[21] K. K. Kahma, "A study of the growth of the wave spectrum with fetch," J. Phys. Oceanogr., vol. 11, pp. 1503-1515, Nov. 1981.

[22] A. K. Fung and K. K. Lee, "A semi-empirical sea-spectrum model for scattering coefficient estimation," IEEE J. Oceanic Eng., vol. OE-7, Oct. 1982.

[23] G. Franceschetti, M. Migliaccio, D. Riccio, and G. Schirinzi, "SARAS: A SAR raw signal simulator," IEEE Trans. Geosci. Remote Sensing, vol. 30, pp. 110-123, Jan. 1992.

[24] G. Franceschetti, M. Migliaccio, and D. Riccio, "SAR raw signal simulation of actual ground sites described in terms of sparse input data," IEEE Trans. Geosci. Remote Sensing, vol. 32, pp. 1160-1169, Nov. 1994.

[25] L. E. Borgman, "Techniques for computer simulation of ocean waves," Rendiconti S.I.F., vol. LXXX, pp. 387-417, 1982.

[26] J. C. West, R. K. Moore, and J. C. Holtzman, "The sligthly-rough facet model in radar imaging of the ocean surface," Int. J. Remote Sens., vol. 11, pp. 617-637, 1990 .

[27] R. O. Harger, "The SAR image of short gravity waves on a long gravity wave," in Wave Dynamics and Radio Probing of the Ocean Surface, O. M. Phillips and K. Hasselmann, Eds. New York: Plenum, 1986, pp. 371-392.

[28] M. Migliaccio, D. Riccio, and G. Schirinzi, "Preliminary considerations for an efficient SAR ocean simulation," ETT, vol. 2, pp. 707-713, 1991.

[29] H. Mitsuyasu and T. Honda, "The effects of surfactants on certain air-sea interaction phenomena," in Wave Dynamics and Radio Probing of the Ocean Surface, O. M. Phillips and K. Hasselmann, Eds., 1997, pp. 95-115.

[30] A. P. Wallenberger and D. R. Lyzenga, "Measurement of the surface tension of water using microwave backscatter from gravity-capillary waves," IEEE Trans. Geosci. Remote Sensing, vol. 28, pp. 1012-1016, Nov. 1990.

[31] W. Alpers et al., "Radar signatures of oil films floating on the sea surface and the Marangoni effect," J. Geophys. Res., vol. 93, pp. 3642-3648, 1988.

[32] M. Gade, W. Alpers, H. Huhnerfuss, and P. A. Lange, "Wind wave tank measurements of wave camping and radar cross sections in the presence of monomolecular surface films," J. Geophys. Res., vol. 103, pp. 3167-3178, 1998.

[33] P. P. Lombardini, F. Piazzese, and R. Cini, "The Marangoni wave ripples on an air-water interface covered by a spreading film," Nuovo Cimento, vol. 5C, no. 2, 1982.

[34] B. Fiscella, P. P. Lombardini, P. Trivero, and R. Cini, "Ripple damping on water surface covered by a spreading film: Theory and experiment," Nuovo Cimento, vol. 8C, no. 5, 1985.

[35] V. G. Levich, Physico-Chemical Hydrodynamics. Englewood Cliffs, NJ: Prentice-Hall, 1962.

[36] J. C. Gottifredi and G. J. Jameson, "The suppression of wind-generated waves by a surface film," J. Fluid Mech., vol. 32, no. 3, pp. 609-618, 1968

[37] R. Cini, P. P. Lombardini, and H. Huhnerfuss, "Remote sensing of marine slicks utilizing their influence on wave spectra," Int. J. Remote Sens., vol. 4, no. 1, pp. 101-110, 1983

[38] M. Gade, W. Alpers, H. Huhnerfuss, H. Masuko, and T. Kobauashi, "Imaging of biogenic and anthropogenic ocean surface films by the multifrequency/multipolarization SIR-C/X-SAR," J. Geophys. Res., vol. 103 , no. 18 , pp. $851-866,1988$.

[39] S. A. Ermakov, A. M. Zujkova, A. R. Panchenko, S. G. Salashin, T. G. Talipova, and V. I. Titov, "Surface film effect on short wind waves," Dynam. Atmos. Oceans, vol. 10, pp. 31-50, 1986.

[40] P. Trivero, B. Fiscella, F. Gomez, and P. Pavese, "SAR detection and characterization of sea surface slicks," Int. J. Remote Sens., vol. 19, pp. 543-548, 1998 
[41] H. Masuko, "Observation of artificial slicks with SIR-C/X SAR around Japan," in Proc. IGARSS, Florence, Italy, July 14-18, pp. 227-229.

[42] M. Gade and J. M. Redondo, "Marine pollution in European coastal waters monitored by the ERS-2 SAR: A comprehensive statistical analysis," in Proc. IGARSS, Hamburg, Germany, pp. 1375-1377.

[43] Hoffmann, H. Huehnerfuss, J. Simon-Kutscher, and W. Alpers, "Morphology of sea slick looking on the sea surface with the 'eye of a chemist'," in Proc. IGARSS, Hamburg, Germany, p. 1469.

[44] K. Litovchenko, "Detection of oil slicks parameters from ALMAZ and ERS-1 SAR imagery," in Proc. IGARSS, Hamburg, Germany, pp. 1484-1486.

[45] G. Franceschetti, Electromagnetics. Theory, Techniques and Engineering Paradigms. New York: Plenum, 1999.

[46] P. W. Vachon and R. K. Raney, "Ocean waves and optimal SAR processing: Don't adjust the focus!," IEEE Trans. Geosci. Remote Sensing, vol. 30, pp. 627-630, May 1992.

[47] F. Del Frate, A. Petrocchi, J. Lichtenegger, and G. Calabresi, "Neural networks for oil spill detection using ERS-SAR data," IEEE Trans. Geosci. Remote Sensing, vol. 38, pp. 2282-2287, Sept. 2000.

[48] G. Benelli and A. Garzelli, "Oil spills detection in SAR images by fractal dimension estimation," in Proc. IGARSS, Hamburg, Germany, pp. 218-220.

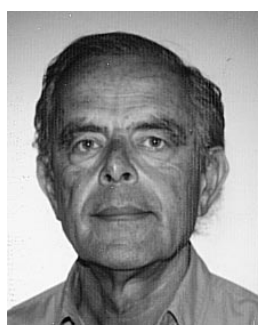

Giorgio Franceschetti (S'60-M'62-SM'85-F'88LF'01) was born in Italy.

He is currently Full Professor of electromagnetic wave theory at University of Naples, Naples, Italy, since 1968. He is also an Adjunct Professor at the University of California, Los Angeles and a Distinguished Visiting Scientist at the Jet Propulsion Laboratory, California Institute of Technology (Caltech), Pasadena. He was a Visiting Professor at the University of Illinois, Urbana-Champaign, in 1976 and 1977, and at the UCLA in 1980 and 1982. He was a Research Associate at Caltech, in 1981 and 1983, a Visiting Professor at National Somalia University, Mogadishu, Somalia, in 1984, and a Visiting Professor at the University of Santiago de Compostela, Santiago de Compostela, Spain, in 1995. He has published several books and more than 130 refereed papers in the field of applied electromagnetics (reflector antennas, transient phenomena, shielding, nonlinear propagation, and scattering) and, more recently, in the field of SAR data processing and simulation. He has lectured in several summer schools in China, the United Kingdom, Holland, Italy, Spain, Sweden, and the United States.

Dr. Franceschetti was a Fulbright Scholar at Caltech in 1973. He has been the recipient of several national and international awards. He was Director of IRECE, a Research Institute of the Italian National Council of Research (CNR), and member of the board of the Italian Space Agency (ASI).

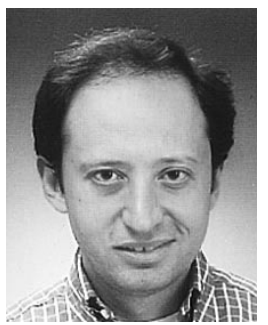

Antonio lodice (S'97-M'00) was born in Naples, Italy, on July 4, 1968. He received the Laurea degree (with honors) in electronic engineering and the Ph.D degree in electronic engineering and computer science, both from the University of Naples "Federico II,” Naples, Italy, in 1993 and 1999, respectively.

$\mathrm{He}$ is currently a Research Scientist at the Department of Electronic and Telecommunication Engineering, University of Naples "Federico II." In 1995, he received a grant from the Italian National Council of Research (CNR) to be spent at Istituto di Ricerca per l'Elettromagnetismo e i Componenti Elettronici (IRECE), Naples, Italy, for research in the field of remote sensing. He was with the Department of Electronic and Telecommunication Engineering of the University of Naples "Federico II" from 1996 to 1999, and with Telespazio S.p.A., Rome, Italy, from 1999 to 2000. His main research interests are in the field of SAR remote sensing: modeling of electromagnetic scattering from natural surfaces and SAR interferometry.

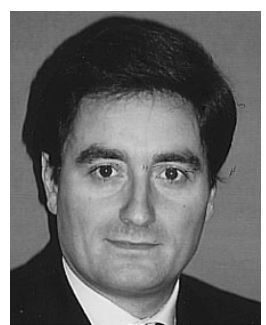

Daniele Riccio (M'91-SM'99) was born in Naples, Italy, on April 13, 1962. He received the Laurea degree (with honors) in electronic engineering from the University of Naples "Federico II," Naples, Italy, in 1989.

Currently, he is Professor of remote sensing, electromagnetic diagnostics, and guided propagation at the University of Naples "Federico II." He was a Research Scientist at the Istituto di Ricerca sull'Elettromagnetismo e i Componenti Elettronici (IRECE), Italian National Council of Research (CNR), and at the Department of Electronic and Telecommunication Engineering, University of Naples "Federico II." In 1994 and 1995, he was also a Guest Scientist at the DLR High-Frequency Institute, Munich, Germany. His main research activities are in the fields of microwave remote sensing and simulation and modeling of SAR signals relevant to terrestrial and oceanic scenes, as well as in the application of fractal geometry to electromagnetic scattering and remote sensing.

Prof. Riccio has won several fellowships from private and public companies (SIP, Selenia, CNR, CORISTA, and CRATI) for research in the remote sensing field.

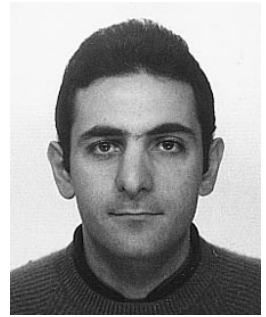

Giuseppe Ruello (S'00) was born in Naples, Italy, on February 12, 1975. He received the Laurea degree (with honors) in telecommunication engineering from the University of Naples "Federico II", Naples, Italy, in 1999, where he is currently pursuing the Ph.d. degree in electronic and telecommunication engineering.

In 2000, he received a grant from the University of Naples to be spent at the Department of Electronic and Telecommunication Engineering for research in the field of remote sensing. In 2000 , he won also a grant from University of Rome "La Sapienza," Rome, Italy. In 2002, he was a Visiting Scientist at the Department of Signal Theory and Communications of the Universitat Politecnica de Catalunya, Barcelona, Spain. His main research interests are in the field of SAR remote sensing, modeling of electromagnetic scattering from natural surfaces, and SAR simulation.

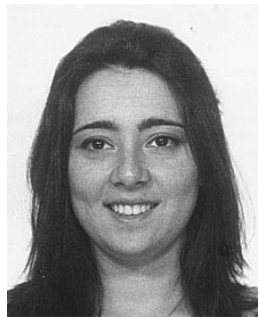

Roberta Siviero was born in Naples, Italy, on July 15, 1977. She attended a training course at Telecom Italia on local loop unboundling. She graduated "summa cum laude" in Telecommunication Engineering at the University of Naples "Federico II," Naples, Italy, in 2001.

Currently, she works for WISE, Pozzuoli, Italy, and her research field is the simulation of SAR signals and electromagnetic field in urban areas. From 2000 to 2001, she was with the Department of Electronic and Telecommunication Engineering of the University of Naples "Federico II," and her research focused on the modeling and simulation of synthetic aperture radar signals related to oceanic surfaces covered by oil films. 